\title{
Listado de la colección de hongos (Ascomycota y Basidiomycota) del Herbario Nacional del Ecuador (QCNE) del Instituto Nacional de Biodiversidad (INABIO)
}

\author{
Rosa Batallas-Molina', Gabriela Fernanda Moya-Marcalla ${ }^{2}$, Daniel Navas Muñoz ${ }^{3}$ \\ ${ }^{1}$ Instituto Nacional de Biodiversidad del Ecuador (INABIO), colección micológica del Herbario \\ Nacional del Ecuador (QCNE), Av. Río Coca E6-115 e Isla Fernandina, sector Jipijapa, Quito, Ecuador \\ 2Programa de Maestría en Biodiversidad y Cambio Climático, Universidad Tecnológica \\ Indoamérica (UTI), Machala y Sabanilla, Quito, Ecuador \\ ${ }^{3}$ Carrera en Ingeniería en Biodiversidad y Recursos Genéticos, Universidad Tecnológica \\ Indoamérica (UTI), Machala y Sabanilla, Quito, Ecuador \\ *Autor para correspondencia / Corresponding author, email: rosa.batallas@biodiversidad.gob.ec
}

\section{Checklist of the fungi collection (Ascomycota and Basidiomycota) of the National Herbarium of Ecuador (QCNE) of the National Institute of Biodiversity (INABIO)}

\begin{abstract}
The National Herbarium of Ecuador (QCNE) of the National Institute of Biodiversity (INABIO) preserves the public mycological collection of Ecuador, being the most representative of the country with 6200 specimens between fungi and lichens. The mycological collection started in 1999 with contributions from university students and specialists who deposited their specimens in the cryptogam section of the QCNE Herbarium. Since 2013 the information has been digitized and 4400 records were taken as the basis for this manuscript. The objective of this work is to present the list of species of fungi of the mycological collection of QCNE. The collection of fungi is organized according to the criteria of the most recent specialized literature, and 319 species of fungi are reported, corresponding to samples from the Ascomycota and Basidiomycota divisions. There are 12 orders, 30 families, 72 genera and 116 species of Ascomycota, with the family Xylariaceae having the highest representativeness (45\%). There are 12 orders, 53 families, 168 genera and 203 species of Basidiomycota, with the family Polyporaceae being the best represented (24\%9. Out of 843 species of fungi registered in Fungi web, QCNE has approximately $32 \%$ of the species reported for the country.
\end{abstract}

Editado por /

Edited by:

Diego F.

Cisneros-Heredia

Recibido /

Received:

12/03/2020

Aceptado /

Accepted:

04/01/2021

Publicado en línea / Published online:

$15 / 04 / 2021$
Keywords: catalogue, fungi, Polyporaceae, public mycological repository, Xylariaceae

\section{Resumen}

El Herbario Nacional del Ecuador (QCNE) del Instituto Nacional de Biodiversidad (INABIO) conserva la colección micológica pública del Ecuador, siendo la más representativa del país con 6200 especímenes entre hongos y líquenes. La colección micológica nació en 1999 con el aporte de colectas de estudiantes universitarios y especialistas que depositaron sus especímenes en la sección de criptógamas del Herbario QCNE. Desde el 2013 la 
información se ha digitalizado y 4400 registros fueron tomados como base para este manuscrito. El objetivo del presente trabajo es presentar el listado de especies de hongos de la colección micológica del QCNE. La colección de hongos se encuentra organizada siguiendo criterios de la literatura especializada más reciente, se reportan 319 especies de hongos correspondientes a muestras de las divisiones Ascomycota y Basidiomycota. Hay 12 órdenes, 30 familias, 72 géneros y 116 especies de Ascomycota, con la familia Xylariaceae teniendo la mayor representatividad (45\%). Hay 12 órdenes, 53 familias, 168 géneros y 203 especies de Basidiomycota, con la familia Polyporaceae siendo la mejor representatada (24\%). De las 843 especies de hongos registradas en Fungi web, el QCNE posee aproximadamente el $32 \%$ de las especies reportadas para el país.

Palabras clave: catálogo, Polyporaceae, repositorio micológico público, Xylariaceae

\section{INTRODUCCIÓN}

Los herbarios constituyen repositorios de material vegetal herborizado que preservan colecciones de muestras botánicas como material científico de referencia para conocer la diversidad florística de una región [1], incluyendo especímenes de plantas no vasculares, hongos y líquenes. Aunque taxonómicamente el Reino Fungi constituye un grupo aparte, usualmente dentro de los herbarios están ubicadas las colecciones micológicas como repositorios de ejemplares herborizados de hongos y hongos liquenizados ó líquenes, cuyo voucher incluye información geográfica, taxonómica, ecológica y descriptiva del espécimen preservado.

El Herbario Nacional del Ecuador (QCNE) fue creado en 1977 como el departamento de Botánica del Museo Ecuatoriano de Ciencias Naturales [2]. Actualmente conforma la sección botánica del Instituto Nacional de Biodiversidad (INABIO), un instituto público de investigación ecuatoriano enfocado en estudios de biodiversidad, que alberga aproximadamente 250000 ejemplares de herbario entre plantas vasculares, no vasculares, hongos y líquenes, de los cuales 180000 están reportados en una plataforma virtual [3]. La colección micológica del QCNE fue creada como parte de la sección de criptógamas, esta iniciativa en 1999 fue liderada por tesistas de pregrado de la Universidad Central del Ecuador quiénes colectaron, procesaron y catalogaron las primeras colecciones de hongos y hongos liquenizados. Este repositorio empezó a crecer gracias al valioso aporte de investigadores como Tatiana Sanjuán [4-5] y estudiantes de pregrado de la Universidad Central del Ecuador, creciendo aceleradamente con el depósito de especímenes de estudiantes e investigadores extranjeros, alcanzando el año 2006 un total 1400 especímenes de los dos grupos.

Desde el año 2010, la colección micológica del QCNE ha participado como contraparte nacional en un proyecto liderado por la Universidad de Oregón, cuyo compromiso fue depositar los especímenes de hongos con etiquetas producto de esta investigación según Autorización de Investigación Científica № 12-10-IC-FLO-DPAI/MA otorgada por el Ministerio del Ambiente Ecuador (MAE), incrementando rápidamente el número de especímenes en la colección. En 2013 el crecimiento de la colección motivó la 
implementación de una base de datos preliminar para almacenar y manejar toda información de la colección micológica, permitiendo un eficiente registro digital de los datos de cada espécimen, ingresando la información del voucher.

En la actualidad, el repositorio científico alberga 6200 especímenes catalogados, correspondiente a una variada y representativa colección de especímenes de hongos (4400) y líquenes (1800) colectados en varias provincias de Ecuador. La colección micológica en cuanto a hongos se incrementa temporalmente con especímenes colectados en investigaciones realizadas por colectores nacionales e internacionales $[6,7]$. Desde sus inicios, el propósito de la colección micológica ha sido conservar y documentar especímenes de hongos y líquenes ecuatorianos, que sirvan como referente en la investigación y difusión de la diversidad fúngica encontrada en el país. Este artículo abarca el grupo de hongos registrando las especies dentro de las divisiones Basidiomycota y Ascomycota que forman parte del patrimonio micológico del sector estatal. Los objetivos del presente trabajo son: difundir el contenido taxonómico y reportar el listado de hongos sistemáticamente organizado del repositorio micológico del Herbario QCNE del INABIO.

\section{MATERIALES Y MÉTODOS}

Para realizar el análisis del contenido micológico y posterior listado de las especies de hongos se analizaron 4400 datos digitalizados, de los cuales se consideraron para el listado, las especies catalogadas e ingresadas en el fichero que fueron determinados por especialistas extranjeros de los herbarios (ASU, CP, FCME, XAL) y de la Universidad de Oregon. En algunos casos se reexaminó las especies con terminología "cf." y "aff." para validar su correcta identificación, mediante el análisis de estructuras microscópicas como esporas, hifas, fíbulas y otras con la ayuda del estereoscopio, el microscopio y el uso de claves taxonómicas y bibliografía especializada [8-22].

Durante la actualización de la taxonomía de los especímenes se revisaron las sinonimias, actualizando géneros y especies mediante la consulta en la plataforma Index Fungorum [23] y para especies nuevas reportadas a partir del 2015 se añadió el código de MykoBank registrado en la página MycoBank Database con las siglas MB [24]. Para organizar la información recopilada tomamos como referencia el siguiente material bibliográfico: Rodríguez et al. [25], Dios et al. [26] y Fernández-Fernández et al. [27].

En el manuscrito la información está organizada alfabéticamente por órdenes, familias, géneros y especies dentro de dos grupos taxonómicos superiores (Ascomycota y Basidiomycota). Para cada especie se presenta información del nombre científico actualizado en la base de Index Fungorum, y el número de catalogación QCNE de la especie. Es importante mencionar que en el caso de especies con varios especímenes se consideró el número de catalogación más antiguo por especie. Se han señalado con un asterisco las especies que se reportan por primera vez para el país en este artículo, según lo reportado en la página Fungi web [28]. Un aspecto importante es la migración de datos del fichero Excel a la plataforma institucional Symbiota proceso que actualmente se va dando paulatinamente. 


\section{RESULTADOS}

En Ecuador, la colección micológica del Herbario Nacional del Ecuador (QCNE) del INABIO constituye el repositorio estatal mejor representado, los resultados de este estudio se centran únicamente en los especímenes de hongos-no se incluye el grupo de líquenes, por consiguiente se reportan 319 especies de hongos, 116 ascomicetes y 203 basidiomicetes. Para Ascomycota se reportan 6 clases, 12 órdenes, 30 familias y 72 géneros; y en Basidiomycota 2 clases, 12 órdenes, 53 familias y 168 géneros. Del total de los datos analizados, 2158 especímenes están determinados hasta género, 421 muestras han sido identificados a nivel de especie y se registran 1821 especímenes indeterminados identificados a nivel de clase, orden o familia. En cuanto al número más alto de especímenes en la colección partiendo de las divisiones Basidiomycota y Ascomycota, se reporta en porcentajes las familias y géneros taxonómicos mejor representados (Tabla 1). Los taxones Auricularia delicata (basidiomicete) y Cookeina tricoloma (ascomicete) constituyen las especies mejor representadas con similar número de registros (14).

TABLA 1. Familias y Géneros reportados en la colección micológica del QCNE-INABIO.

\begin{tabular}{|c|c|c|c|c|c|c|c|}
\hline \multicolumn{3}{|c|}{ Basidiomycota } & \multicolumn{5}{c|}{ Ascomycota } \\
\hline Familias & $\%$ & Géneros & $\%$ & Familias & $\%$ & Géneros & $\%$ \\
\hline Polyporaceae & 24 & Polyporus & 14 & Xylariaceae & 45 & Xylaria & 31 \\
\hline Marasmiaceae & 11 & Marasmius & 10 & Cordycipitaceae & 8 & Cookeina & 7 \\
\hline Tricholomataceae & 9 & Stereum & 7 & Sarcoscyphaceae & 6 & Cordyceps & 6 \\
\hline Stereaceae & 5 & Mycena & 6 & Pyronemataceae & 2.5 & Hypoxylon & 4 \\
\hline Hymenochaetaceae & 4 & Auricularia & 4 & Otras familias & 38.5 & Otros géneros & 52 \\
\hline Otras familias & 47 & géneros & 59 & & & & \\
\hline
\end{tabular}

Las colecciones de muestras holotipo y paratipo se encuentran dentro de la colección de tipos del Herbario QCNE, donde se encuentran tres holotipos y tres paratipos. Se reporta 9 especies con su respectivo código de MykoBank, de las cuales 7 constituyen actualizaciones taxonómicas y 2 son nuevas especies para Ecuador, información incluida en el total de especies del manuscrito. 


\section{Listado de holotipos y paratipos depositados en la colección de hongos del Herbario Nacional del Ecuador (QCNE) \\ REINO FUNGI}

\section{DIVISIÓN ASCOMYCOTA}

*Beauveria diapheromeriphila (T. Sanjuan \& S. Restrepo) T. Sanjuan, B. Shrestha, Kepler \& Spatafora, IMA Fungus 8(2): 345 (2017). [MB\#820882]. Holotipo. ORELLANA: Cantón Aguarico. Tiputini field station. 13-julio-2004. T. Laessoe, J. H Petersen y Alsgard Jensen 11390. QCNE186272.

*Ophiocordyceps tiputini Sanjuan, Fungal Biology 119(10): 912 (2015). [MB\#805228]. Holotipo. ORELLANA: Cantón Aguarico. Tiputini field station. 13-julio-2004. T. Laessoe 11465. QCNE186287.

*Thamnomyces chocoënsis Læssøe. Mycoscience 51(3): 200 (2010). [MB\#513277]. Holotipo. PICHINCHA: Cantón San Miguel de los Bancos. Hostería El Encanto vía los Bancos - Santo Domingo, sobre la orilla del rio blanco. 10-agosto-2004. T. Laessoe, C. Padilla, T. Sanjuán y M. Villegas 11800. QCNE186554.

*Beauveria acridophila (T. Sanjuan \& S. Restrepo) T. Sanjuan, B. Shrestha, Kepler \& Spataora, IMA Fungus 8(2): 343 (2017). [MB\#820883]. Paratipo. SUCUMBIOS: Cantón Shushufindi. Limoncocha. Reserva Biológica Limoncocha. 19-septiembre-2004. M. Villegas y C. Padilla 2509. QCNE186726.

*Beauveria locustiphila (Henn.) B. Shrestha, Kepler \& Spatafora. IMA Fungus 8(2): 345 (2017). [MB\#820884]. Paratipo. ORELLANA: Cantón: Aguarico. Tiputini field station. 17-julio-2004. T. Laessoe y Mayer 11514. QCNE186267.

*Ophiocordyceps fulgoromorphila Sanjuan, Fungal Biology 119(10): 911 (2015). [MB\#805227]. Paratipo. ORELLANA: Cantón: Aguarico. Yasuní National Park. Tiputini field station. 13-julio-2004. T. Laessoe 11466. QCNE186286.

\section{Listado de la colección de hongos del Herbario Nacional del Ecuador (QCNE)}

\section{DIVISIÓN ASCOMYCOTA}

\section{SORDARIOMYCETIDAE}

Lasiosphaeriella nitida Huhndorf \& F.A. Fernández, Mycologia 91 (3): 545 (1999). ORELLANA: Cantón Aguarico. Yasuní National Park, Perú Trail. 6-Marzo-2001. Fernández, A.N. Miller y R. Briones 4365. QCNE152142.

Lasiosphaeriella pseudobombarda (Mont.) Huhndorf \& F.A. Fernández, Mycologia 91(5): 922 (1999). ORELLANA: Cantón Aguarico. Yasuní National Park, Perú Trail. 4-Marzo-2001. Fernández, A.N. Miller y R. Briones 4370. QCNE152143.

\section{SORDARIOMYCETES}

Stromatographium stromaticum (Berk.) Höhn. Ergebth. Bot. Exp. Sudakras Deut. k. Ak. d. Wiss Wien 83:37 (extr.) (1907). IMBABURA: Cantón Cotacachi. García Moreno. Reserva Los Cedros. 24-abril-2014. R. Vandegrift y D. Newman 1296. QCNE201422. 


\section{BOLINIALES \\ BOLINIACEAE}

Camarops ustulinoides (Henn.) Nannf. Svensk Botanisk Tidskrift. 66 (4): 370 (1972). ZAMORA CHINCHIPE: Cantón Zamora. Parroquia El Tambo. Estación Científica San Francisco carretera Loja - Zamora. 28-marzo-2002. D. Suárez 1003. QCNE152129.

\section{CHAETOSPHARIALES}

\section{CHAETOSPHAERIACEAE}

Sporoschisma hemipsilum (Berk. \& Broome) Zelski, A.N. Mill. \& Shearer, IMA Fungus 5 (2): 433 (2014). ORELLANA: Cantón Aguarico. Yasuní National Park, Chorongo Trail. 9-Marzo-2001. Fernández, A.N. Miller y R. Briones 4401. QCNE152148.

*Striatosphaeria codinaeaphora Samuels \& E. Müll., Sydowia 31 (1-6): 132 (1979). ORELLANA: Cantón Aguarico. Yasuní National Park, along road near Ceiba tree. 8-Marzo-2001. Fernández, A.N. Miller y R. Briones 4398. QCNE152176.

\section{GEOGLOSSALES}

GEOGLOSSACEAE

Sarcoleotia globosa (Sommerf.) Korf, Phytologia 21(4): 206 (1971). NAPO: Cantón Quijos. Parroquia Papallacta, páramo de la Virgen. 2-agosto-2004. T. Laessoe y K. Hansen 11747. QCNE 186446.

\section{HELOTIALES CHLOROCIBORIACEAE}

Chlorociboria aeruginascens (Nyl.) Kanouse ex C.S. Ramamurthi, Korf \& L.R. Batra, Mycologia 49: 858 (1958). ZAMORA CHINCHIPE: Cantón Zamora. Parroquia El Tambo. Estación Científica San Francisco carretera Loja - Zamora. 28-marzo-2002. D. Suárez 997. QCNE152105.

\section{HELOTIACEAE}

Bisporella citrina (Batsch) Korf \& S.E. Carp., Mycotaxon 1 (1): 42 (1974). PICHINCHA: Cantón Quito. Bosque Protector Pichincha. Sector Bosque Cochapamba. 3-mayo-2003. C. Padilla, T. Sanjuán y R. Batallas 529. QCNE152760.

\section{HEMIPHACIDIACEAE}

*Encoelia cubensis (Berk. \& M.A. Curtis) Iturr., Samuels \& Korf. Mycotaxon 52(1): 272 (1994). ZAMORA CHINCHIPE: Cantón Zamora. Parroquia El Tambo. Estación Científica San Francisco carretera Loja - Zamora. 28-marzo-2002. D. Suárez 1023a. QCNE152228.

*Encoelia cf. heteromera (Mont.) Nannf., Trans. Br. mycol. Soc. 23(3): 239 (1939). ORELLANA. Cantón Francisco de Orellana. Inés Arango. Cerca de Barneno a orillas del río Cononaco, territorio Huahorani. 17-agosto-2004. L. Guzmán 9207. QCNE186203. 


\section{HYALOSCYPHACEAE}

Hyaloscypha aureliella (Nyl.) Huhtinen, Karstenia 29 (2): 107 (1990). PICHINCHA: Cantón Quito. Volcán Pichincha, la Primavera N, side of quebrada. 21-julio-2003. T. Laessoe, K. Hansen, J. H Petersen y Alsgard Jensen 11544. QCNE186559.

\section{HYPOCREALES \\ CLAVICIPITACEAE}

*Conoideocrella luteorostrata (Zimm.) D. Johnson, G.H. Sung, Hywel-Jones \& Spatafora, Mycological Research 113 (3): 286 (2008). IMBABURA: Cantón Cotacachi. García Moreno. Reserva Los Cedros. 20-junio-2010. M. Wherley, J. McAlpine y B. Roy 219. QCNE201903. Nigelia martiale (Speg.) Luangsa-ard \& Thanakitp., Mycological Progress 16 (4): 380 (2017). SUCUMBIOS: Cantón Shushufindi. Limoncocha. Reserva Biológica Limoncocha. 19-septiembre-2004. M. Villegas y C. Padilla 2498. QCNE152406.

\section{CORDYCIPITACEAE}

*Akanthomyces aculeatus Lebert, Z. Wiss. Zool. 9: 449 (1858). ORELLANA: Cantón: Aguarico. Tiputini field station. 17-julio-2004. T. Laessoe TL-A. QCNE201160.

Akanthomyces tuberculatus (Lebert) Spatafora, Kepler \& B. Shrestha, IMA Fungus 8(2): 343 (2017). [MB\#820863]. CARCHI: Cantón Espejo. Zona de amortiguamiento de la Reserva Ecológica El Angel, relictos de bosque de Weimmannia. 14-octubre-2004. T. Sanjuán, M. Villegas y R. Batallas 523. QCNE152203.

Ascopolyporus villosus Möller, Botanische Mittheilungen aus den Tropen 9: 301 (1901). IMBABURA: Cantón Cotacachi. García Moreno. Reserva Los Cedros. 18-enero-2008. B. Dentingen y T. Jenkinson 110. QCNE201901.

*Cordyceps farinosa (Holmsk.) Kepler, B. Shrestha \& Spatafora, IMA Fungus 8(2): 346 (2017)). [MB\#820979]. PICHINCHA: Cantón Pedro Vicente Maldonado. Km 105 via Pachijal, finca la nueva esperanza de Elly Richardson. 30-mayo-2003. T. Sanjuán y C. Padilla 394. QCNE186096.

${ }^{*}$ Cordyceps neogryllotalpae Kobayasi, Bull. natn. Sci. Mus., Tokyo, B 2(4): 143 (1976). SUCUMBIOS: Cantón Shushufindi. Parroquia Limoncocha. Reserva Biológica Limoncocha, sendero Pishira. 19-septiembre-2004. M. Villegas y C. Padilla 2498. QCNE186720.

Cordyceps nidus T. Sanjuán, Chir-Salom. \& S. Restrepo, PloS ONE 12 (6): e0179428, 7 (2017). [MB\#817601]. IMBABURA: Cantón Cotacachi. García Moreno. Reserva Los Cedros. 01-enero-2019. N. Dueñas 11. QCNE246402.

*Cordyceps polyarthra Möller, Bot. Mitt. Trop. 9: 213 (1901). ORELLANA: Cantón: Aguarico. Tiputini field station. 17-julio-2004. T. Laessoe, Sanjuán y C. Padilla 11335. QCNE152163.

${ }^{*}$ Cordyceps pruinosa Petch, Transactions of the British Mycological Society 10 (1-2): 38 (1924). IMBABURA: Cantón Cotacachi, García Moreno, Reserva Los Cedros. 24-diciembre-2018. P. Goyes, J. Flores y R. Vandegrift 12. QCNE246403.

${ }^{*}$ Cordyceps pseudonelumboides Kobayasi \& Shimizu, Bulletin of the National Science Museum Tokyo 8 (3): 80 (1982). SUCUMBIOS: Cantón Shushufindi. Limoncocha. Reserva Biológica Limoncocha. 17-septiembre-2004. M. Villegas y C. Padilla 2508. QCNE201461. ${ }^{*}$ Cordyceps takaomontana PYakush. Kumaz. Sci. Rep. Tokyo Bunrika Daig. Sectec. B 5: 108 (1941). IMBABURA: Cantón Cotacachi, García Moreno, Reserva Los Cedros. 25-diciembre-2018. Wolk 14. QCNE246406. 
Cordyceps tenuipes (Peck) Kepler, B. Shrestha \& Spatafora, IMA Fungus 8 (2): 347 (2017). PICHINCHA: Cantones: Quito y Sto Domingo. Bosque Protector Río Guajalito (QUSF). 14-octubre-2004. R. Batallas, M. Villegas, C. Padilla y R. Leiva 822. QCNE152206.

\section{HYPOCREACEAE}

*Trichoderma lixii (Pat.) P. Chaverri, Jaklitsch, Gazis, Degenkolb \& Samuels, Mycologia 107(3): 578 (2015). IMBABURA: Cantón Cotacachi. García Moreno. Reserva Los Cedros. 18-enero-2008. B. Dentingen y T. Jenkinson 110. QCNE201901.

\section{NECTRIACEAE}

Nectria cf. cinnabarina (Tode) Fr., Summa veg. Scand., Sectio Post. (Stockholm): 388 (1849). ORELLANA: Cantón: Aguarico. Tiputini field station, sendero harpia. 13-julio-2004. T. Laessoe, J.H. Petersen, T. Sanjuán y C. Padilla 11467. QCNE152153.

*Neocosmospora solani (Mart.) L. Lombard \& Crous, Stud. Mycol. 80: 228 (2015). ORELLANA: Cantón: Aguarico. Tiputini field station, sendero guacamayo. 13-julio-2004. T. Sanjuán, C. Padilla, T. Laessoe y J.H. Petersen 473b. QCNE152154.

\section{OPHIOCORDYCIPITACEAE}

Hirsutella fusiformis Speare, Mycologia 12(2): 70 (1920). PICHINCHA: Cantón Pedro Vicente Maldonado. Km 105 via Pachijal, finca la Nueva Esperanza de Elly Richardson. 30-mayo2003. T. Sanjuán y C. Padilla 395. QCNE186051.

Ophiocordyceps amazonica Henn, Studies in Mycology 57: 40 (2007). QCNE152202.

SUCUMBIOS: Cantón Shushufindi. Limoncocha. Reserva Biológica Limoncocha. 17-septiembre-2004. M. Villegas y C. Padilla 2499. QCNE152202.

Ophiocordyceps australis (Speg.) G.H. Sung, J.M. Sung, Hywel-Jones \& Spatafora, Studies in Mycology 57: 40 (2007). ORELLANA: Cantón Aguarico. Yasuní National Park, Perú trail. 10-Marzo-2001. Fernández, A.N. Miller y R. Briones 4432. QCNE152107.

Ophiocordyceps caloceroides (Berk. \& M.A. Curtis) Petch, Trans. Br. mycol. Soc. 18(1): 63 (1933). ORELLANA: Cantón: Aguarico. Tiputini field station. 14-julio-2004. T. Laessoe, J.H. Petersen y A. Alsgard Jensen TL-C. QCNE201379.

Ophiocordyceps curculionum (Tul. \& C. Tul.) G.H. Sung, J.M. Sung, Hywel-Jones \& Spatafora, Studies in Mycology. 57: 41 (2007). NAPO: Cantón Tena. Estación Biológica Jatun Sacha a 8 $\mathrm{Km}$ al este de Misahualli al sur del rio Napo. 20-septiembre-2002. R. Batallas, A. Mariscal y L. Parra 342. QCNE152809.

Ophiocordyceps dipterigena (Berk. \& Broome) G.H. Sung, J.M. Sung, Hywel-Jones \& Spatafora, Studies in Mycology 57: 42 (2007). PICHINCHA: Cantón San Miguel de los Bancos. Hostería El Encanto vía los Bancos - Santo Domingo, sobre la orilla del rio blanco. 10-agosto-2004. T. Laessoe, C. Padilla, T. Sanjuán y M. Villegas 11802. QCNE186694.

Ophiocordyceps gracilioides Kobayasi, Science Reports of the Tokyo Bunrika Daigaku 5: 140 (1941). ORELLANA: Cantón: Aguarico. Tiputini field station. 18-julio-2004. T. Laessoe, J.H. Petersen y A. Alsgard Jensen TL-B. QCNE201366.

*Ophiocordyceps melolonthae Tul. \& C. Tul.) G.H. Sung, J.M. Sung, Hywel-Jones \& Spatafora, Studies in Mycology 57: 44 (2007). PICHINCHA: Cantón Quito. Nanegal, zona Gualea, Finca Mapali. 10-agosto-2001. W. Chamorro, D. Montalvo, A. Luzuriaga y M. Ortega 128. QCNE152207. 
Ophiocordyceps nutans (Pat.) G.H. Sung, J.M. Sung, Hywel-Jones \& Spatafora, Studies in Mycology 57: 45 (2007). ORELLANA: Cantón: Aguarico. Tiputini field station. 14-julio-2004. T. Laessoe y J.H. Petersen. TL-E. QCNE186500.

*Ophiocordyceps sphecocephala (Klotzsch. Ex Berk.) G.H. Sung, Hywel-Jones \& Spatafora, Stud. Mycol. 57: 47 (2007). IMBABURA: Cantón Cotacachi. García Moreno. Reserva Los Cedros, oso trail. 01-enero-2019. N. Dueñas 24. QCNE246415.

Ophiocordyceps unilateralis (Tul. \& C. Tul.) Petch, Trans. Br. mycol. Soc. 16(1): 74 (1931). NAPO: Cantón Tena. Estación Biológica Jatun Sacha a 8 Km de puerto Misahualli margen derecha del rio Napo. 29-septiembre-2002. R. Batallas, A. Mariscal y L. Parra 404. QCNE186013.

*Tolypocladium cf. inflatum W. Gams, Persoonia 6(2): 185 (1971). PICHINCHA: Cantón San Miguel de los Bancos. Hostería El Encanto vía los Bancos - Santo Domingo, sobre la orilla del rio blanco. 10-agosto-2004. T. Sanjuán, T. Laessoe, M. Villegas y R. Batallas 503. QCNE186141.

\section{JOBELLICIALES}

JOBELLICIACEAE

Jobellisia barrii Huhndorf, Lodge \& F.A. Fern., Sydowia 51 (2): 186 (1999). ORELLANA: Cantón Aguarico. Yasuní National Park, Garza Trail. 5-marzo-2001. F.A. Fernández, A.N. Miller y R. Briones 4351. QCNE152130.

\section{BOLINIALES}

\section{PEZIZALES}

\section{CALOSCYPHACEAE}

*Caloscypha fulgens (Pers.) Boud, Hist. Class. Discom. Eur. (Paris): 54 (1907). CARCHI: San Pedro de Huaca. Parroquia Mariscal Sucre. 25-agosto-2004. V. Jácome 86. QCNE186894.

\section{MORCHELLACEAE}

Morchella esculenta (L.) Pers., Syn. meth. fung. (Göttingen) 2: 618 (1801). PICHINCHA: Cantón Quito. Parroquia Lloa, Palmira sector quebrada "Baños". 16-abril-2003. K. Escobar y A. Luzuriaga 100. QCNE201301.

\section{PEZIZACEAE}

*Adelphella babingtonii (Berk. \& Broome) Pfister, Matočec \& I. Kušan, Mycologia Montenegrina 11: 14 (2009) [2008]. NAPO: Cantón Quijos. Parroquia Papallacta, shrub along small river above "aguas termales". 22-julio-2004. T. Laessoe, K. Hansen, J.H. Petersen y A. Alsgard 11580. QCNE201343.

\section{PYRONEMATACEAE}

*Aleuria aurantia (Pers.) Fuckel, Jb. nassau. Ver. Naturk. 23-24: 325 (1870) [1869-70]. PICHINCHA: Cantón Quito. Parroquia Calacalí, Sector Yunguilla "La Liberia". 14-julio-2001. N. Osorio 53. QCNE 201197 
Scutellinia scutellata (L.) Lambotte, Mém. Soc. roy. Sci. Liège, Série 2 14: 299 [prepr.] (1887) [1888]. PICHINCHA: Cantón Quito. Bosque Protector Pichincha. Sector Bosque Rosa de los Andes. 10-mayo-2003. T. Sanjuán, C. Padilla y R. Batallas 373. QCNE152759.

\section{SARCOSCYPHACEAE}

Cookeina speciosa (Fr.) Dennis, Mycotaxon 51: 239 (1994). AZUAY: Cantón Molleturo. 9-marzo-2001. Ernesto Muela 1. QCNE152109.

Cookeina tricholoma (Mont.) Kuntze, Revis. gen. pl. (Leipzig) 2: 849 (1891). NAPO: Cantón Tena. Parque Amazónico. 3-mayo-1999. D.Suárez 134. QCNE152106.

*Cookeina cf. venezuelae (Berk. \& M.A. Curtis) Le Gal, Discomyc. Madagascar (Paris): 239 (1953). PICHINCHA: Cantón San Miguel de los Bancos. Parroquia Mindo. Hacienda la Espontánea cerca del rio Mindo. D. Suárez 232. QCNE152115.

Phillipsia domingensis (Berk.) Berk. Ex Denison, Mycologia 61(2): 293 (1969). IMBABURA: Cantón Cotacachi, García Moreno. Reserva Los Cedros. 16-enero-2008. B. Dentingen y T. Jenkinson 82. QCNE201902.

*Wynnea americana Thaxt., Bot. Gaz. 39: 241 (1905). IMBABURA: Cantón Cotacachi. García Moreno. Reserva Los Cedros. 18-enero-2011. B. Dentingen, T. Jenkinson y J. McAlpine 831. QCNE201915.

Wynnea gigantea Berk. \& M.A. Curtis, J. Linn. Soc. Bot. 9: 424 (1867). QCNE244529.

IMBABURA: Cantón Cotacachi. García Moreno. Reserva Los Cedros. 30-abril-2014. D. Newman y S. Boden. QCNE244529.

\section{PLEOSPORALES MELANOMMATACEAE}

Byssosphaeria jamaicana (Sivan.) M.E. Barr, Mycotaxon 20(1): 30 (1984). ORELLANA: Cantón Aguarico, Yasuní National Park, Perú Trail. 6-marzo-2001. F. A. Fernández, A.N. Miller y R. Briones 4363. QCNE152079.

Byssosphaeriaschiedermayeriana (Fuckel) M.E. Barr, Mycotaxon 20(1):34 (1984). ORELLANA: Cantón Aguarico. Yasuní National Park, Tinamu Trail. 5-marzo-2001. F.A. Fernández, A.N. Miller y R. Briones 4342. QCNE152080.

\section{SORDARIALES \\ LASIOSPHAERIACEAE}

Lasiosphaeria ellisii M.E. Barr, Mycotaxon 46: 48 (1993). ORELLANA: Cantón Aguarico, Yasuní National Park, Garza Trail. 5-marzo-2001. F.A. Fernández, A.N. Miller y R. Briones 4352. QCNE152093.

Lasiosphaeria rufula (Penz. \& Sacc.) Rossman, Mycologia 69(2): 375 (1977). ORELLANA: Cantón Aguarico. Yasuní National Park, Bariso Trail. 7-marzo-2001. F.A. Fernández, A.N. Miller y R. Briones 4377. QCNE152144.

Lasiosphaeria rugulosa (A.N. Mill. \& Huhndorf) A.N. Mill. \& Huhndorf, Mycol. Res. 108(1): 31 (2004). ORELLANA: Cantón Aguarico. Yasuní National Park, Perú Trail. 5-marzo-2001. F.A. Fernández, A.N. Miller y R. Briones 4341. QCNE152093. 


\section{XYLARIALES \\ GRAPHOSTROMATACEAE}

Biscogniauxia cf. capnodes var. capnodes (Berk.) Y.M. Ju \& J.D. Rogers, Mycotaxon 66: 23 (1998). NAPO: Cantón Quijos. Parroquia Papallacta, sendero el arriero. Polylepis forest. 2-agosto-2004. T. Laessoe y K. Hansen 11750. QCNE186374.

Camillea leprieurii (Mont.) Mont., Summa veg. Scand., Sectio Post. (Stockholm): 382 (1849). NAPO: Cantón Tena, Estación Biológica Jatun Sacha a 8 Km al este de Misahualli al sur del rio Napo. 5-septiembre-2002. R. Batallas, A. Mariscal y L. Parra 261. QCNE186104.

*Camillea stellata Læssøe, J.D. Rogers \& Whalley, Mycol. Res. 93(2): 144 (1989). ORELLANA: Cantón: Aguarico. Tiputini field station. 14-julio-2004.T. Laessoe, J.H. Petersen y A. Alsgard Jensen 11425. QCNE18654.

Camillea cf. venezuelensis (J.H. Mill.) Dennis, Kew Bull., Addit. Ser. 3: 296 (1970). ORELLANA: Cantón: Aguarico. Tiputini field station. 18-julio-2004.T. Laessoe, J.H. Petersen y A. Alsgard Jensen 11528. QCNE186328.

*Theissenia pyrenocrata (Theiss.) Maubl. Bull. Soc. mycol. Fr. 30(1): 52 (1914). ORELLANA: Cantón: Aguarico. Tiputini field station. 16-julio-2004. T. Laessoe, J.H. Petersen y A. Alsgard Jensen 11480. QCNE186421.

\section{HYPOXYLACEAE}

*Daldinia concentrica_(Bolton) Ces. \& De Not., Comm. Soc. crittog. Ital. 1 (fasc. 4): 197 (1863). SUCUMBIOS: Cantón: Cuyabeno. Tarapora. Comunidad Siona de Sototsiaya. 15-mayo2005. X. Arturo 48. QCNE186914.

Jackrogersella multiformis (Fr.) L. Wendt, Kuhnert \& M. Stadler, Mycological Progress 17 (1-2): 138 (2017). NAPO: Cantón Quijos. Parroquia Papallacta, Bellow Papallacta, just above Goango. 2-agosto-2004. T. Laessoe y K. Hansen 11556. QCNE186165.

Phylacia poculiformis (Mont.) Mont., Ann/s Sci. Nat. Bot., sér. 4 3: 135 (1855). ORELLANA: Cantón Aguarico. Yasuní National Park, Garza Trail. 5-marzo-2001. F.A. Fernández, A.N. Miller y R. Briones 4350. QCNE152161.

*Phylacia cf. surinamensis (Berk. \& M.A. Curtis) Dennis, Kew Bull. 12 (2): 325 (1957). NAPO: Cantón Tena. Estación Biológica Jatun Sacha a $8 \mathrm{Km}$ al este de Misahualli al sur del rio Napo. 11-septiembre-2002. R. Batallas, A. Mariscal y L. Parra 323. QCNE186037.

*Pyrenopolyporus nicaraguensis (Ellis \& Everh.) M. Stadler, Kuhnert \& L. Wendt, Mycological Progress 17 (1-2): 150 (2017). QCNE186930. GALÁPAGOS: Santa Cruz. Los Gemelos zona de Scalesia. 24-agosto- 2005. Xavier Arturo 56. QCNE186930.

Thamnomyces chordalis Fr., Linnaea 5: 534 (1830). PICHINCHA: Cantón San Miguel de los Bancos. Hostería El Encanto vía los Bancos - Santo Domingo, sobre la orilla del rio blanco. 10-agosto-2004. T. Laessoe, C. Padilla, T. Sanjuán y M. Villegas 11801. QCNE186555

*Thamnomyces dendroideus Cooke \& Massee, Grevillea 16 (79): 69 (1888). PICHINCHA: Cantón San Miguel de los Bancos. Hostería El Encanto vía los Bancos - Santo Domingo, sobre la orilla del rio blanco. 10-agosto-2004. T. Laessoe, C. Padilla, T. Sanjuán y M. Villegas 11435. QCNE186256.

\section{XYLARIACEAE}

*Kretzschmaria cf. cetrarioides (Welw. \& Curr.) Sacc., Syll. fung. (Abellini) 2: XXIX (1883). ORELLANA: Cantón Aguarico. Yasuní National Park, Garza Trail. 5-marzo-2001. F.A. Fernández, A.N. Miller y R. Briones 4349. QCNE152131. 
Kretzschmaria clavus (Fr.) Sacc., Syll. fung. (Abellini) 2: XXIX (1883). ORELLANA: Cantón: Aguarico. Tiputini field station. 13-julio-2004. T. Laessoe, J.H. Petersen y A. Alsgard Jensen 11394. QCNE186472.

*Kretzschmaria lucidula (Mont.) Dennis, Kew Bull., Addit. Ser. 3: 300 (1970). IMBABURA: Cantón Cotacachi. García Moreno. Reserva Los Cedros. 6-enero-2012. B. Roy, D. Thomas y D. Simba 922. QCNE201921.

Kretzschmaria macrosperma (Mont.) J.D. Rogers \& Y.M. Ju, Mycotaxon 68: 358 (1998). PICHINCHA: Cantón Quito, río Blanco afluente del río Cinto. 9-agosto-2002. M. Díaz, L. Pineida y O. Taimal 11. QCNE186199.

*Kretzschmaria of. pavimentosa (Ces.) P.M.D. Martin, JI S. Afr. Bot. 42(1): 74 (1976) ORELLANA: Cantón Aguarico. Tiputini field station. 17-julio-2004. T. Laessoe, J.H. Petersen y A. Alsgard Jensen 11498. QCNE186332.

Nemania bipapillata (Berk. \& M.A. Curtis) Pouzar, Ceská Mykologie 39 (1): 24 (1985). IMBABURA: Cantón Cotacachi. García Moreno. Reserva Los Cedros. 18-mayo-2014. R. Vandegrift y D. Newman 1429. QCNE201942.

*Nemania diffusa (Sowerby) Gray, Nat. Arr. Brit. Pl. (London) 1: 517 (1821). CARCHI: Cantón San Pedro de Huaca. Parroquia Mariscal Sucre. 25-julio-2004. T. Laessoe, J.H. Petersen y C. Cárdenas-Batallas 11668. QCNE186515.

Stilbohypoxylon moelleri Henn., Hedwigia 41: 16 (1902). ORELLANA: Cantón Aguarico. Yasuní National Park, along road near chorongo tree. F.A. Fernández, A.N. Miller y R. Briones 4411. QCNE152175.

*Xylaria allantoidea (Berk.) Fr., Nova Acta R. Soc. Scient. upsal. Ser. 3 1(1): 127 (1851) [1855]. IMBABURA: Cantón Cotacachi. García Moreno. Reserva Los Cedros. 11-mayo-2014. R. Vandegrift y D. Newman 1446. QCNE201941.

*Xylaria anisopleura (Mont.) Fr., Nova Acta R. Soc. Scient. upsal. Ser. 3 1 (1): 127 (1851) [1855]. IMBABURA: Cantón Cotacachi. García Moreno. Reserva Los Cedros. 7-enero-2012. B. Roy, D. Thomas y R. Vandegrift 936. QCNE201922.

Xylaria apiculata Cooke, Grevillea 8(46): 66 (1879). IMBABURA: Cantón Cotacachi. García Moreno. Reserva Los Cedros. 23-abril-2014. R. Vandegrift y D. Newman 1313. QCNE201937.

*Xylaria atrosphaerica (Cooke \& Massee) Callan \& J.D. Rogers, Mycotaxon 36(2): 349 (1990). IMBABURA: Cantón Cotacachi. García Moreno. Reserva Los Cedros. 6-enero-2012. B. Roy y D. Thomas 907. QCNE201919.

Xylaria cf. coccophora Mont., Annls Sci. Nat., Bot., sér. 4 3: 109 (1855). ORELLANA: Cantón Aguarico. Yasuní National Park. 6-marzo-2001. F.A. Fernández, A.N. Miller y R. Briones 4356. QCNE152180.

Xylaria comosoides Læssøe, Kew Bull. 54(3): 611 (1999). NAPO: Cantón Tena. Estación Biológica Jatun Sacha a $8 \mathrm{Km}$ de Puerto Misahualli margen derecha del Rio Napo. 10-enero-2002. D. Suárez y M. Asanza. Informantes: Pedro Grefa y Angel Alvarado 985. QCNE152214.

Xylaria cubensis (Mont.) Fr., Nova Acta R. Soc. Scient. upsal. Ser. 3 1(1): 126 (1851) [1855]. Anamorph: Xylocoremium flabeliforme (Schwein.) J.D. Rogers, Mycologia 76(5): 914 (1984). PICHINCHA: Cantón Quito, suroriente SE of Nanegalito, Km 49, Puma Sacha, cascada del rayo. T. Laessoe, J.H. Petersen, C.A. Padilla y R. Batallas 11336. QCNE 186349.

Xylaria cuneata Lloyd, Mycol. Writ. 7(Letter 68): 1180 (1923). IMBABURA: Cantón Cotacachi. García Moreno. Reserva Los Cedros. 7-junio-2012. B. Roy, D. Thomas y D. Simba 954. QCNE201926. 
Xylaria aff. curta Fr., Nova Acta R. Soc. Scient. upsal. Ser. 3 1(1): 126 (1851) [1855]. IMBABURA: Cantón Cotacachi. García Moreno. Reserva Los Cedros. 5-enero-2012. B. Roy y D. Thomas 897. QCNE201916.

Xylaria enterogena Mont., Syll. gen. sp. crypt. (Paris): 203 (1856). IMBABURA: Cantón Cotacachi. García Moreno. Reserva Los Cedros. 21-enero-2010. M. Wherley, J. McAlpine y B. Roy 311. QCNE201913.

Xylaria fissilis Ces., Atti Accad. Sci. fis. mat. Napoli 8(3): 16 (1879). IMBABURA: Cantón Cotacachi. García Moreno. Reserva Los Cedros. 25-enero-2010. M. Wherley, J. McAlpine y B. Roy 448. QCNE201906.

*Xylaria fuscopurpurea Laessøe \& Lodge, The Food Web of a Tropical Rain Forest, Chicago: 87 (1996). PICHINCHA: Cantón San Miguel de Los Bancos. Parroquia Mindo. Reserva Mindo Lindo. Km 60 de la via Calacalí. 4-febrero-2001. E. Muela y R. Batallas 7. QCNE152211.

Xylaria guianensis (Mont.) Fr., Nova Acta R. Soc. Scient. upsal. Ser. 3 1(1): 127 (1851) [1855]. NAPO: Cantón Tena. Estación Biológica Jatun Sacha. 11-septiembre-2002. R. Batallas, A. Mariscal y L. Parra 331. QCNE186044.

Xylaria hyperythra (Mont.) Mont., Syll. gen. sp. crypt. (Paris): 202 (1856). ORELLANA: Cantón Aguarico. Tiputini field station. 13-julio-2004. T. Laessoe, J.H. Petersen y A. Alsgard Jensen, C.A. 11405. QCNE186264.

Xylaria hypoxylon (L.) Grev., Fl. Edin.: 355 (1824). PICHINCHA: Cantón Quito. Gualea Cruz, parche A, sureste 35 de Gualea Cruz. 6-agosto-2000. D. Suárez, G. Pérez y M. Arévalo 279. QCNE152184.

*Xylaria kegeliana (Lév.) Fr., Nova Acta R. Soc. Scient. upsal. Ser. 3 1(1): 125 (1851) [1855]. IMBABURA: Cantón Cotacachi, García Moreno. Reserva Los Cedros. 26-abril-2014. R. Vandegrift 1245. QCNE201934.

*Xylaria melanura (Lév.) Sacc., Syll. fung., Addit. I-IV (Abellini): 403 (1886). IMBABURA: Cantón Cotacachi. García Moreno. Reserva Los Cedros. 22-enero-2010. M. Wherley, J. McAlpine y B. Roy 375. QCNE201912.

Xylaria meliacearum Læssøe, Mycologia 86(3): 441 (1994). IMBABURA: Cantón Cotacachi, García Moreno. Reserva Los Cedros. 9-enero-2012. B. Roy, D. Thomas y D. Simba 959. QCNE201931.

Xylaria mellissii (Berk.) Cooke, Grevillea 11 (59): 85 (1883). PICHINCHA: Cantón San Miguel de Los Bancos. Parroquia Mindo. Reserva Mindo Lindo. Km 60 de la via Calacali. 4-febrero-2001. R. Batallas 12. QCNE152189.

*Xylaria microceras (Mont.) Berk., Nova Acta R. Soc. Scient. upsal. Ser. 3 1(1): 128 (1851) [1855]. ORELLANA: Cantón: Aguarico. Tiputini field station. 16-julio-2004. T. Laessoe, J.H. Petersen y A. Alsgard Jensen 11388. QCNE247557.

Xylaria multiplex (Kunze) Fr., Nova Acta R. Soc. Scient. upsal. Ser. 3 1(1): 127 (1851) [1855]. IMBABURA: Cantón Cotacachi. García Moreno. Reserva Los Cedros. 26-enero-2010. F. Bechtolsheim y J. McAlpine 508. QCNE201909.

*Xylaria cf. nodulosa Lloyd, Mycol. Writ. 6(Letter 64): 1007 (1920) [1921]. SUCUMBIOS: Cantón: El Dorado de Cascales. Campo Bermejo. 1-mayo-2001. D. Suárez 403. QCNE152190.

Xylaria obovata (Berk.) Berk., Nova Acta R. Soc. Scient. upsal. Ser. 3 1(1): 127 (1851) [1855]. PICHINCHA: Cantón Santo Domingo de los Colorados. Estación Biológica Gustavo Orcés, km 11 1/2 vía Quevedo. 4-diciembre-1999. D. Suárez, G. Pérez y M. Trujillo 208b. QCNE152216. 
Xylaria ophiopoda Sacc., Annls mycol. 4(1): 74 (1906). IMBABURA: Cantón Cotacachi. García Moreno. Reserva Los Cedros. 6-enero-2012. B. Roy y D. Thomas 911. QCNE201920. Xylaria palmicola G. Winter, Grevillea 15 (75): 89 (1887). ORELLANA: Cantón: Aguarico. Tiputini field station. 17-julio-2004. T. Laessoe, J.H. Petersen y A. Alsgard Jensen 11503. QCNE186308.

Xylaria schweinitzii Berk. \& M.A. Curtis, J. Acad. nat. Sci. Philad., N.S. 2(6): 284 (1854) [1853]. IMBABURA: Cantón Cotacachi. García Moreno. Reserva Los Cedros. 5-enero-2012. B. Roy y D. Thomas 880. QCNE201946.

Xylaria scruposa (Fr.) Fr., Nova Acta R. Soc. Scient. upsal. Ser. 3 1(1): 127 (1851) [1855]. PICHINCHA: Cantón Santo Domingo de los Colorados. Sector finca "la Chamarra", via Sto. Domingo-Quininde km 16. Rosa Batallas 268. QCNE152200.

*Xylaria subtorulosa Speg., Boln Acad. Nac. Cienc. Córdoba 11(4): 515 (1889). IMBABURA: Cantón Cotacachi. García Moreno. Reserva Los Cedros. 9-enero-2012. B. Roy, D. Thomas, D. Simba, R. Vandegrift 1026. QCNE201923.

Xylaria telfairii (Berk.) Sacc., Sylloge Fungorum 1: 320 (1882). ORELLANA: Estación IAMOE 65 km al sur del Coca. Vía Pindo-Auca. 27-julio-2000. Jean Quezada Lucero. QCNE152192. Xylaria tuberoides Rehm, Hedwigia 40: 146 (1901). PICHINCHA: Cantón Quito. Nanegal. Zona Gualea, Finca Mapali. 22-febrero-2001. W. Chamorro, D. Montalvo, L. Luzuriaga y M. Ortega 42. QCNE152118.

*Xylaria venosula Speg., Boln Acad. Nac. Cienc. Córdoba 11(4): 511 (1889). IMBABURA: Cantón Cotacachi. García Moreno. Reserva Los Cedros. 7-enero-2012. B. Roy, D. Thomas y R. Vandegrift 942. QCNE201924.

Xylosphaera comosa (Mont.) Dennis, Kew Bull. [13](1): 103 (1958). PICHINCHA: Cantón Quito. Gualea Cruz, parche A, sureste $35^{\circ}$ de Gualea Cruz. D. Suárez y M. Arévalo 257. QCNE152868.

Xylosphaera xanthinovelutina (Mont.) Dennis, Kew Bull. [13](1): 104 (1958). ORELLANA: Cantón: Aguarico. Tiputini field station. 16-julio-2004. T. Laessoe, J.H. Petersen y A. Alsgard Jensen 11484. QCNE186313.

\section{XYLOBOTRIALES \\ XYLOBOTRIACEAE}

*Xylobotryum andinum Pat. Bull. Herb. Boissier 3(1): 69 (1895). IMBABURA: Cantón Cotacachi. García Moreno. Reserva Los Cedros. 29-diciembre-2018. Oblando y Lomas 31. QCNE246401.

Xylobotryum portentosum (Mont.) Pat., Bull. Soc. mycol. Fr. 16(4): 185 (1900). IMBABURA: Cantón Cotacachi. García Moreno. Reserva Los Cedros. 17-abril-2014. R. Vandegrift 1277. QCNE201420.

Xylobotryum rickii (Theiss.) Lloyd. Mycol. Writ. 7(Letter 75): 1350 (1925). SUCUMBIOS: Cantón Gonzalo Pizarro. Lumbaquí, dentro del derecho de vía del OCP. 28-mayo-2002. D. Suárez, S. Endara y C. Morales 1056. QCNE152227.

\section{DIVISIÓN BASIDIOMYCOTA}

AGARICOMYCETES

*Cotylidia aurantiaca (Pat.) A.L, Welden, Lloydia 21: 40 (1958). PICHINCHA: Cantón Quito. Nanegal. Zona Gualea, Finca Mapali. 22-febrero-2001. W. Chamorro, D. Montalvo, L. Luzuriaga y M. Ortega 45. QCNE152279. 
Cotylidia diaphana (Cooke) Lentz, Agric. Monogr. U.S.D.A. 24: 12 (1955). IMBABURA: Cantón Cotacachi. García Moreno. Reserva Los Cedros. 15-diciembre-2018. D. Newman QCNE201612.

\section{AGARICALES \\ AGARICACEAE}

*Agaricus minor J.F. Gmel., Syst. Nat., Edn 13 2(2): 1398 (1792). CARCHI: Cantón San Pedro de Huaca. Parroquia Mariscal Sucre. Estación Biológica Guandera. 25-julio-2004. T. Laessoe, J.H. Petersen y C. Cárdenas-Batallas, K. 11645. QCNE186508.

*Agaricus sylvaticus Schaeff., Fung. bavar. palat. nasc. (Ratisbonae) 4:62 (1774). PICHINCHA: Cantón Quito. Bosque Protector Pichincha. Sector Bosque Cochapamba. 3-mayo-2003.T. Sanjuán, C. Padilla y R. Batallas 365. QCNE152232.

*Battarrea phalloides (Dicks.) Pers., Syn. meth. fung. (Göttingen) 1: xiv, 129 (1801). PICHINCHA: Cantón Pedro Moncayo. Malchinguí. Bosque Protector Jerusalem, sendero avifauna. 7-mayo-2006. R. Batallas y A. Troya 1138. QCNE201080.

Bovista plumbea Pers., Ann. Bot. (Usteri) 15: 4 (1795). NAPO: Cantón Quijos. Papallacta. Reserva Ecológica Antisana. 12-febrero-1999. D. Suárez, J. Quezada 123. QCNE201060.

*Chlorophyllum molybdites (G. Mey.) Masse., Bull. Misc. Inf., Kew: 136 (1898). MANABÍ: Cantón Puerto López, Mantaraya Lodge 2 km south of Puerto López. 29-julio-2004. T. Laessoe, K. Hansen, J.H Petersen y A. Alsgard Jensen. 11702. QCNE186469.

Crucibulum laeve (Huds.) Kambly, Gast. lowa: 167 (1936). PICHINCHA: Cantón Quito. Bosque Protector Pichincha. Sector Bosque de Cochapamba. 3-mayo-2003. T. Sanjuán, C. Padilla y R. Batallas 365. QCNE152873.

${ }^{*}$ Cystolepiota luteohemisphaerica (Dennis) I. Saar \& Læssøe, Mycotaxon 104: 317 (2008) MANABÍ: Cantón Puerto López, Rio Ayampe near the village. 31-julio-2004. T. Laessoe, K Hansen, J.H Petersen y A. Alsgard 11724. QCNE186439.

Cystolepiotapulverulenta (Huijsman) Vellinga, Persoonia 14 (4): 407 (1992). CARCHI: Cantón San Pedro de Huaca. Parroquia Mariscal Sucre. Estación Biológica Guandera. 3-mayo2003. T. Laessoe, K. Hansen, J.H Petersen y A. Alsgard Jensen 11643. QCNE186525.

*Leucoagaricus sulphurellus (Pegler) B.P. Akers, Mycotaxon 76: 48 (2000). MANABÍ: Cantón Puerto López, Mantaraya Lodge 2 km south of Puerto López. 29-julio-2004. T. Laessoe, K. Hansen, J.H Petersen y A. Alsgard Jensen. 11713. QCNE186391.

*Leucocoprinus fragilissimus (Ravenel ex Berk. \& M.A. Curtis) Pat., Essai Tax. Hyménomyc. (Lons-le-Saunier): 171 (1900). MANABÍ: Cantón Puerto López, Mantaraya Lodge 2 km south of Puerto López. 29-julio-2004. T. Laessoe, K. Hansen, J.H Petersen y A. Alsgard Jensen. 11704. QCNE186386.

Lycogalopsis solmsii E. Fisch., Ber. dt. bot. Ges. 4: 197 (1886). NAPO: Cantón Tena. Estación Biológica Jatun Sacha. 11-septiembre-2002. R. Batallas, A. Mariscal y L. Parra 325. QCNE186039. Lycoperdon perlatum Pers., Observ. mycol. (Lipsiae) 1: 4 (1796). PICHINCHA: Cantón Quito, Nono. Reserva Orquideológica Pahuma, sendero Oreja de Mono. 14-diciembre-2002. R. Martínez y D. Barrera 27. QCNE152359.

*Lycoperdon pisiforme Henn., Monsunia 1: 158 (1899) [1900]. CARCHI: Cantón San Pedro de Huaca. Parroquia Mariscal Sucre. Estación Biológica Guandera. 17-abril -2009. A. Toapanta 01. QCNE244856.

Melanophyllum haematospermum (Bull.) Kreisel, Feddes Repert. Spec. Nov. Regni Veg. 95(910): 700 (1984). ORELLANA: Cantón: Aguarico. Tiputini field station. 17-julio-2001. T. Laessoe, K. Hansen, J.H. Petersen y A. Alsgard Jensen 11509. QCNE186333. 
Nidula macrocarpa Lloyd, Mycol. Writ. 5(Letter 51): 731 (1917). NAPO: Cantón Quijos. Just below Papallacta pass, paramo El Virgen. 11-julio-2004. T. Laessoe, J.H. Petersen y A. Alsgard Jensen 11363. QCNE186531.

\section{BOLBITIACEAE}

Rhodoarrhenia cyphelloides (Lloyd) Singer, Sydowia 17(1-6): 143 (1964). IMBABURA: Cantón Cotacachi. García Moreno. Reserva Los Cedros. 16-abril-2014. R. Vandegrift y D. Newman 1234. QCNE201421.

\section{CLAVARIACEAE}

*Clavaria aff. taxophila Lloyd, Mycol. Writ. 6(Letter 64): 1008 (1920) [1921]. CARCHI: Cantón San Pedro de Huaca. Parroquia Mariscal Sucre. Estación Biológica Guandera. 25-julio-2004. T. Laessoe, J.H. Petersen y C. Cárdenas-Batallas, K. 11687. QCNE186523.

${ }^{*}$ Clavulinopsis aff. antillarum (Pat.) Courtec, Docums Mycol. 33(131): 36 (2004). PICHINCHA: Cantón San Miguel de los Bancos. Parroquia Mindo. Los Bancos km 79 de la carretera Calacalí-La Independencia. Bosque Protector Mindo Lindo. 31-julio-2003. D. Suárez 843. QCNE201684.

*Clavulinopsis laeticolor (Berk. \& M.A. Curtis) R.H. Petersen, Mycologia 57(4): 522 (1965). ZAMORA CHINCHIPE: Cantón Zamora. El Tambo. Estación Científica San Francisco carretera Loja-Zamora. 28-marzo-2002. D. Suárez 1006. QCNE152262.

*Ramariopsis minutula (Bourdot \& Galzin) R.H. Petersen, Mycologia 58(2): 202 (1966). PICHINCHA. Bosque Protector Río Guajalito, sendero los españoles. Km 59 de la antigua vía a Santo Domingo de los Colorados.20-diciembre-2003. G. Martínez 6. QCNE186751.

\section{CYPHELLACEAE}

Campanophyllum proboscideum (Fr.) Cifuentes \& R.H. Petersen, Mycol. Progr. 2(4): 289 (2003). CARCHI: Cantón San Pedro de Huaca. Parroquia Mariscal Sucre. Estación Biológica Guandera. 23-julio-2004. T. Laessoe, J. H Petersen y T. Sanjuán 11600. QCNE186482.

\section{ENTOLOMATACEAE}

*Entoloma dunstervillei (Dennis) E. Horak, Sydowia 30(1-6): 511977 (1978). IMBABURA: Cantón Cotacachi. García Moreno. Reserva Los Cedros. 12-junio-2008. B. Dentingen y T. S. Jenkinson 19. QCNE201352.

\section{HYDNANGIACEAE}

Laccaria amethystina Cooke, Grevillea 12(63): 70 (1884). CARCHI: Cantón San Pedro de Huaca. Parroquia Mariscal Sucre. Estación Biológica Guandera. 7-diciembre-2002. C. Cárdenas y C. Padilla 22. QCNE152696.

Laccaria fraterna (Sacc.) Pegler, Aust. J. Bot., Suppl. Ser. 13(2): 332 (1965). PICHINCHA: Cantón Quito. Bosque Protector Pichincha, SE de Quito, loma el Ungui, Ciudadela Tarqui. 7-diciembre-2002. R. Batallas, C. Padilla y I. Jalca 534. QCNE152341. 
*Laccaria laccata (Scop.) Cooke, Grevillea 12(63): 70 (1884). PICHINCHA: Cantón Quito. Bosque Protector Pichincha, Sector Bosque de Cochapamba. 13-abril-2005. C. Rivadeneira-Roura y J. Salazar 55. QCNE186965.

\section{HYGROPHORACEAE}

*Hygrocybe coccinea (Schaeff.) P. Kumm., Führ. Pilzk. (Zerbst): 112 (1871). PICHINCHA: Cantón Quito. Nanegal. Zona Gualea, Finca Mapali. 22-febrero-2001. W. Chamorro, D. Montalvo, L. Luzuriaga y M. Ortega 32. QCNE152333.

Hygrocybe conica (Schaeff.) P. Kumm., Führ. Pilzk. (Zerbst): 111 (1871). PICHINCHA: Cantón Quito. Bosque Protector Pichincha, Sector Bosque de Cochapamba. 21-abril-2002. T. Sanjuán 235. QCNE201503.

*Hygrocybe hypohaemacta (Corner) Pegler, Kew Bull. 32(2): 299 (1978). IMBABURA: Cantón Cotacachi. García Moreno. Reserva Los Cedros. 20-enero-2010. M. Wherley, J. McAlpine y B. Roy 272. QCNE201373.

Hygrocybe miniata (Fr.) P. Kumm., Führ. Pilzk. (Zerbst): 112 (1871). ZAMORA CHINCHIPE: Cantón Zamora. El Tambo. Estación Científica San Francisco carretera Loja-Zamora. 28-marzo-2002. D. Suárez 100. QCNE152332.

\section{HYMENOGASTRACEAE}

Gymnopilus lepidotus Hesler, Mycol. Mem. 3: 40 (1969). IMBABURA: Cantón Cotacachi. García Moreno. Reserva Los Cedros. 13-junio-2008. B. Dentinger y T. S. Jenkinson 41. QCNE201356.

Psilocybe cubensis (Earle) Singer, Sydowia 2(1-6): 37 (1948). ESMERALDAS: Cantón Muisne. Muisne - Monpiche, carretera hacia la casa del prefecto. 19-julio-2002. T. Sanjuán 531. QCNE186163.

*Psilocybe plutonia (Berk. \& M.A. Curtis) Sacc., Syll. fung. (Abellini) 5: 1056 (1887). IMBABURA: Cantón Cotacachi. García Moreno. Reserva Los Cedros. 31-junio-2008. B. Dentinger y T. S. Jenkinson 130. QCNE201367.

*Psilocybe subzapotecorum Guzmán, Docums Mycol. 29(116): 46 (2000). PICHINCHA: Cantón Quito. Nono. Reserva Orquideológica Pahuma, sendero Oreja de Mono. 14-diciembre-2002. R. Martínez y D. Barrera 32. QCNE152989.

*Psilocybezapotecorum R. Heim, RevueMycol., Paris 22:77 (1957).PICHINCHA:Cantón Quito. Nono. Reserva Orquideológica Pahuma, sendero Oreja de Mono. 14-diciembre-2002. R. Martínez y D. Barrera 22. QCNE152739.

\section{INOCYBACEAE}

*Crepidotus eucalypticola Singer, Nova Hedwigia 29 (1-2): 73 (1977). PICHINCHA: Cantón Quito. Bosque Protector Pichincha, Sector Bosque de Cochapamba. 03-mayo-2003. T. Sanjuán 597. QCNE201548.

${ }^{*}$ Crepidotus pallidus (Berk. \& Broome) Knudsen, Cladistics 26: 67 (2010). ORELLANA: Cantón: Aguarico. Tiputini field station. 17-julio-2004. T. Laessoe, K. Hansen, J.H. Petersen y A. Alsgard Jensen 11513. QCNE186322.

*Crepidotus variabilis (Pers.) P. Kumm., Führ. Pilzk. (Zerbst): 74 (1871). CARCHI: Cantón San Pedro de Huaca. Parroquia Mariscal Sucre. Estación Biológica Guandera. 29-diciembre-2004. V. Jácome 84. QCNE201434. 


\section{LYOPHYLLACEAE}

*Lyophyllum decastes (Fr.) Singer, Lilloa 22: 165 (1951) [1949]. LOJA: Cantón Saraguro. Bosque protegido por comunidades kichwas "Washapamba". 1-agosto-2003. S.Villamar 58. QCNE152369.

\section{MARASMIACEAE}

Marasmius aciculiformis Berk. \& M.A. Curtis. J. Linn. Soc., Bot. 10(45): 297 (1868) [1869]. PICHINCHA: Cantón San Miguel de los Bancos. Parroquia Mindo. Santuario de las cascadas. 15-julio-2001. R. Batallas 168. QCNE152369.

Marasmius berteroi (Lév.) Murrill, N. Amer. Fl. (New York) 9(4): 267 (1915). PICHINCHA: Cantón Quito. Nanegal. Zona Gualea, Finca Mapali. 22-febrero-2001. W. Chamorro, D. Montalvo, L. Luzuriaga y M. Ortega 14. QCNE152370.

Marasmius cladophyllus Berk., Hooker's J. Bot. Kew Gard. Misc. 8: 138 (1856). PICHINCHA: Cantón Quito. Nono. Reserva Orquideológica Pahuma, sendero Oreja de Mono. 14-diciembre-2002. R. Martínez y D. Barrera 38. QCNE 152756.

Marasmius crinis-equi F. Muell. Ex Kalchbr. Grevillea 8(48): 153 (1880).IMBABURA: Cantón Cotacachi. García Moreno. Reserva Los Cedros. 13-junio-2008. B. Dentinger y T. S. Jenkinson 41. QCNE201356.

*Marasmius aff. oreades (Bolton) Fr., Anteckn. Sver. Ätl. Svamp.: 52 (1836). PICHINCHA: Cantón Quito. Nanegal. Zona Gualea, Finca Mapali. 10-agosto-2001. W. Chamorro, D. Montalvo, L. Luzuriaga y M. Ortega 111. QCNE152367.

*Marasmius cf. plicatulus Peck, Bull. Torrey bot. Club 24: 142 (1897). PICHINCHA: Cantón Quito. Nanegal. Zona Gualea, Finca Mapali. 22-febrero-2001. W. Chamorro, D. Montalvo, L. Luzuriaga y M. Ortega 13. QCNE152308.

Marasmius siccus (Schwein.) Fr., Epicr. syst. mycol. (Upsaliae): 382 (1838) [1836-1838]. PICHINCHA: Cantón Santo Domingo de los Colorados. Sector finca "La Chamarra", vía Sto. Domingo-Quininde Km 16. 19-enero-2002. R. Batallas 259. QCNE 152375.

*Nothopanus candidissimus (Sacc.) Kühner, Bull. mens. Soc. linn. Lyon 49(Num. Spéc.): 895 (1980). IMBABURA: Cantón Cotacachi, Sector Plaza Guitiérrez. Intag. Protector San Luis de Toabuche.17-julio-2001. N. Chasiliquin y A. Troya 014. QCNE152361.

Tetrapyrgos alba (Berk. \& M.A. Curtis) E. Horak, Sydowia 39: 101 (1987) [1986]. MORONA SANTIAGO: Cantón Morona. San Isidro. Parque Nacional Sangay, Bosque Domono. 25-agosto- 2005. K. Villavicencio y J. Gualotuña 45. QCNE186932.

*Tetrapyrgos nigripes (Fr.) E. Horak, Sydowia 39: 102 (1987). ORELLANA: Cantón: Aguarico. Tiputini field station. 16-julio-2004. T. Laessoe, K. Hansen, J.H. Petersen y A. Alsgard Jensen 11469. QCNE186314.

Trogia cantharelloides (Mont.) Pat., Essai Tax. Hyménomyc. (Lons-le-Saunier): 129 (1900). ORELLANA: Cantón: Aguarico. Tiputini field station. 13-julio-2004. T. Laessoe, K. Hansen, J.H. Petersen \& A. Alsgard Jensen 113959. QCNE186275.

*Trogia icterina (Singer) Corner, Monogr. Cantharelloid Fungi: 218 (1966). IMBABURA: Cantón Cotacachi. García Moreno. Reserva Los Cedros. 9-enero-2012. R. Dentinger, T.S. Jenkinson y J. McAlpine 814. QCNE201385. 
*Favolaschia calocera R. Heim, Revue Mycol., Paris 31 (2): 154 (1966). QCNE186298.

ORELLANA: Cantón: Aguarico. Tiputini field station. 16-julio-2004.T. Laessoe, J.H. Petersen y A. Alsgard Jensen 11473. QCNE186298

Filoboletus gracilis (Klotzsch ex Berk.) Singer, Lloydia 8(3): 216 (1945). IMBABURA: Cantón Cotacachi. García Moreno. Reserva Los Cedros. 12-junio-2008. R. Dentinger, T.S. Jenkinson 3. QCNE201347.

*Hydropus cavipes (Pat. \& Gaillard) Dennis, Kew Bull., Addit. Ser. 3: 42 (1970). IMBABURA: Cantón Cotacachi. García Moreno. Reserva Los Cedros. 5-febrero-2008. B. Dentinger y T. S. Jenkinson 144. QCNE201369.

Hydropus nigrita (Berk. \& M.A. Curtis) Singer, Beih. Sydowia 7: 55 (1973). IMBABURA: Cantón Cotacachi. García Moreno. Reserva Los Cedros. 30-abril-2014. R. Vandegrift y D. Newman 1286. QCNE201419.

Mycena acicula (Schaeff.) P. Kumm., Führ. Pilzk. (Zerbst): 109 (1871). PICHINCHA: Cantón Quito. Bosque Protector Pichincha. Sector Bosque Rosa de los Andes. 10-mayo-2003. T. Sanjuán, C. Padilla y R. Batallas 374. QCNE 152426.

Mycena alcalina (Fr.) P. Kumm. Führ. Pilzk. (Zerbst): 109 (1871). PICHINCHA: Cantón Santo Domingo de los Colorados. Sector finca "La Chamarra", vía Sto. Domingo-Quininde Km 16. 19-enero-2002. R. Batallas 263. QCNE 152434.

Mycena aff. alphitophora (Berk.) Sacc, Syll. fung. (Abellini) 5: 290 (1887). QCNE247883.

IMBABURA: Cantón Cotacachi. García Moreno. Reserva Los Cedros. 23-abril-2014. R. Vandegrift y D. Newman 1293. QCNE247883.

Mycena citricolor (Berk. \& M.A. Curtis) Sacc., Syll. fung. (Abellini) 5: 263 (1887). CARCHI: Cantón San Pedro de Huaca. Parroquia Mariscal Sucre. Estación Biológica Guandera. 23-julio-2004. T. Laessoe, J.H Petersen y T. Sanjuán 11603. QCNE186485.

*Mycena chloroxantha Singer, Cryptog. Mycol. 4(1): 114 (1983). IMBABURA: Cantón Cotacachi. García Moreno. Reserva Los Cedros. 29-diciembre-2018. Oblando y Lomas 38. QCNE246441.

Mycena filopes (Bull.) P. Kumm., Führ. Pilzk. (Zerbst): 110 (1871). CARCHI: Cantón San Pedro de Huaca. Parroquia Mariscal Sucre. Estación Biológica Guandera. 29-diciembre -2004. V. Jácome 93C. QCNE201436.

Mycena holoporphyra (Berk. \& M.A. Curtis) Singer, Sydowia 15(1-6): 64 (1962) [1961]. CARCHI: Cantón San Pedro de Huaca. Parroquia Mariscal Sucre. Estación Biológica Guandera. 29-diciembre -2004. V. Jácome 95. QCNE201437.

*Mycena cf. luteopallens (Peck) Peck, Bull. N.Y. St. Mus. nat. Hist. 1(no. 2): 12 (1887). CARCHI: Cantón San Pedro de Huaca. Parroquia Mariscal Sucre. Estación Biológica Guandera. 29-diciembre -2004. V. Jácome 92. QCNE201431.

*Mycena maculata P. Karst., Meddn Soc. Fauna Flora fenn. 16: 89 (1890) [1889]. PICHINCHA: Cantón Quito. Calacalí. Reserva Geobotánica Pululahua, sendero el chivo. 27-agosto-2004. M. F. Villamil 74. QCNE186901.

Mycena pura (Pers.) P. Kumm., Führ. Pilzk. (Zerbst): 107 (1871). IMBABURA: Cantón Cotacachi. García Moreno. Reserva Los Cedros. 12-junio-2008. R. Dentinger,T.S. Jenkinson 5. QCNE201349. *Mycena sanguinolenta (Alb. \& Schwein.) P. Kumm., Führ. Pilzk. (Zerbst): 108 (1871). CARCHI: Cantón San Pedro de Huaca. Parroquia Mariscal Sucre. Estación Biológica Guandera. 29-diciembre -2004. V. Jácome 82. QCNE186895.

*Panellus luteus (Dennis) Singer, Beih. Sydowia 7: 32 (1973). SUCUMBIOS: Cantón Dorado de Cascales. Campo Bermejo. 29-abril-2001. D. Suárez 380. QCNE152440. 
Panellus pusillus (Pers. ex Lév.) Burds. \& O.K. Mill., Beih. Nova Hedwigia 51: 85 (1975). PICHINCHA: Cantón Quito. Bosque Protector Pichincha, loma el Ungui, Ciudadela Tarqui. 7-diciembre-2002. R. Batallas, C. Padilla y I. Jalca 533. QCNE152301.

Resinomycena saccharifera (Berk. \& Broome) Redhead, Can. J. Bot. 62 (9): 1850 (1984).

ORELLANA: Cantón: Aguarico. Tiputini field station. 18-julio-2004. T. Laessoe, J.H. Petersen y A. Alsgard Jensen 11527. QCNE186293.

Xeromphalina tenuipes (Schwein.) A.H. Sm., Pap. Mich. Acad. Sci. 38: 84 (1953). SUCUMBIOS: Cantón Sushufindi. Limoncocha. Reserva Biológica Limoncocha. 17-septiembre-2004. M. Villegas y C. Padilla 2490. QCNE201174.

\section{OMPHALOTACEAE}

*Gymnopus androsaceus (L.) Della Maggiora \& Trassinelli, Index Fungorum 171: 1 (2014). SUCUMBIOS: Cantón Dorado de Cascales. Campo Bermejo. 21-abril-2001. D. Suárez 384. QCNE152366.

Gymnopus montagnei (Berk.) Redhead, Index Fungorum 148: 1 (2014). SUCUMBIOS: Cantón: Gonzalo Pizarro. Lumbaquí. Dentro del derecho de vía del OCP. 18-mayo-2002. D. Suárez, S. Endara y C. Morales 1043. QCNE152992.

Gymnopus macropus Halling, Brittonia 48(4): 490 (1996). AZUAY: Cantón: Cuenca. Parque Nacional Cajas, sector laguna a Llaviuvu, sendero turístico. 25-octubre-2005. G. F. Brâulete 84. QCNE201334.

*Marasmiellus candidus (Fr.) Singer, Pap. Mich. Acad. Sci. 32: 129 (1948) [1946]. CARCHI: Cantón San Pedro de Huaca. Parroquia Mariscal Sucre. Estación Biológica Guandera. 21-marzo-2009. D. Medina 87. QCNE244831.

Marasmiellus volvatus Singer, Fieldiana Botany 21: 37 (1989). ORELLANA: Cantón: Aguarico. Tiputini field station. 17-julio-2001. T. Laessoe, K. Hansen, J.H. Petersen y A. Alsgard Jensen 11493. QCNE186327.

*Lentinula edodes (Berk.) Pegler, Kavaka 3: 20 (1976). CARCHI: Cantón San Pedro de Huaca. Parroquia Mariscal Sucre. Estación Biológica Guandera. 27-julio-2004. T. Laessoe, J.H Petersen y C. Cárdenas-Batallas 11641. QCNE186411.

*Rhodocollybia amica J.L. Mata \& Halling, Mycol. Progr. 3(4): 339 (2004). IMBABURA: Cantón Cotacachi. García Moreno. Reserva Los Cedros. 22-junio-2008. B. Dentinger y T. S. Jenkinson 119. QCNE201366.

\section{PHYSALACRIACEAE}

Armillaria puiggarii Speg., Boln Acad. nac. Cienc. Córdoba 11(4): 384 (1889). IMBABURA: Cantón Cotacachi. García Moreno. Reserva Los Cedros. 14-junio-2008. B. Dentinger y T. S. Jenkinson 53-A. QCNE201357.

*Flammulina callistosporioides (Singer) Singer, Darwiniana 13: 183 (1964). CARCHI: Cantón San Pedro de Huaca. Parroquia Mariscal Sucre. Estación Biológica Guandera. 25-julio-2004. T. Laessoe, J.H Petersen y C. Cárdenas-Batallas, K. 11641. QCNE186412.

Gloiocephala epiphylla Massee, Grevillea: 34 (1892). IMBABURA: Cantón Cotacachi, García Moreno, Reserva Los Cedros. 18-junio-2008. R. Vandegrift y D. Newman 1510. QCNE201364.

Oudemansiella canarii (Jungh.) Höhn., Sitzungsberichte der Kaiserlichen Akademie der Wissenschaften Math.-naturw. Klasse Abt. I 118: 276 [2 repr.] (1909). ORELLANA: Cantón: Aguarico. Tiputini field station. 18-julio-2004. T. Laessoe, K. Hansen, J.H. Petersen y A. Alsgard Jensen 11538. QCNE186324. 


\section{PLEUROTACEAE}

*Hohenbuehelia angustata (Berk.) Singer, Lilloa 22: 255 (1951). IMBABURA: Cantón Cotacachi. García Moreno. Reserva Los Cedros. 18-junio-2008. B. Dentinger y T. S. Jenkinson 96. QCNE201431.

Pleurotus djamor (Rumph. ex Fr.) Boedijn, Rumphius Memorial Volume: 292 (1959). NAPO: Cantón Tena. Estación Biológica Jatun Sacha, a 8 Km al este de Misahualli, al margen derecho del río Napo 11-septiembre-2002. D. Suárez y S. Villamarín 975. QCNE152476.

\section{PLUTEACEAE}

*Pluteus laetifrons (Berk. \& M.A. Curtis) Sacc., Syll. fung. (Abellini) 5: 677 (1887). NAPO: Cantón El Chaco, N of town at small crossing. 2-agosto -2004. D T. Laessoe y K. Hansen 11774. QCNE201386.

*Pluteus romellii (Britzelm.) Sacc., Syll. fung. (Abellini) 11: 44 (1895). IMBABURA: Cantón Cotacachi. García Moreno. Reserva Los Cedros. 13-junio-2008. B. Dentinger y T. S. Jenkinson 40. QCNE201355.

\section{POROTHELEACEAE}

*Phloeomana speirea (Fr.) Redhead, Index Fungorum 15: 2 (2013). PICHINCHA: Cantón Santo Domingo de los Colorados. Sector finca“La Chamarra", vía Sto. Domingo-Quininde Km. 19-enero-2002. R. Batallas 263. QCNE152434.

\section{PSATHYRELLACEAE}

Coprinellus disseminatus (Pers.) J.E. Lange, Dansk bot. Ark. 9(no. 6): 93 (1938). IMBABURA: Cantón Cotacachi. García Moreno. Reserva Los Cedros. 22-enero-2010. B. Roy, M. Wherley y J. McAlpine 372. QCNE241572.

Coprinellus micaceus (Bull.) Vilgalys, Hopple \& Jacq. Johnson, Taxon 50 (1): 234 (2001). PICHINCHA: Cantón Quito. Bosque Protector Pichincha. Sector Bosque Cochapamba. 3-mayo-2003. T. Sanjuán, C. Padilla y R. Batallas 364. QCNE152297.

${ }^{*}$ Coprinopsis nivea (Pers.) Redhead, Vilgalys \& Moncalvo, Taxon 50(1): 229 (2001). PICHINCHA: Cantón Quito. Bosque Protector Pichincha. Sector Bosque de Cochapamba. 3-mayo-2003. T. Sanjuán, C. Padilla y R. Batallas 356. QCNE152296.

*Panaeolus acuminatus (P. Kumm.) Quél., Mémoires de la Sociétéd'Émulation de Montbéliard Sér. 2, 5: 257 (1872). PICHINCHA: Cantón Quito. Calacalí. Reserva Geobotánica Pululahua. 24-agosto-2004. M. F. Villamil 61. QCNE201442.

Panaeolus papilionaceus (Bull.) Quél., Mém. Soc. Émul. Montbéliard, Sér. 2 5: 152 [122 repr.] (1872). PICHINCHA: Cantón Quito. El Batán. Parque Metropolitano de Quito, sendero de trote sentido norte-sur. 14-diciembre-2002. D. E. Zambrano 26. QCNE201386.

Panaeolus semiovatus (Sowerby) S. Lundell \& Nannf., Fungi Exsiccati Suecici Fasc. 11-12: no. 537 (1938). COTOPAXI: Cantón Sigchos. Parroquia Huacusi, Huacusi cerca del rio Toachi. 12-agosto-1998. D. Suárez 11. QCNE201014.

*Psathyrella corrugis (Pers.) Konrad \& Maubl., Encyclop. Mycol. (Paris) 14: 123 (1949) [1948]. PICHINCHA: Cantón Quito. El Batán. Parque Metropolitano de Quito, sendero de trote sentido este-oeste. 14-diciembre-2002. D. E. Zambrano 22. QCNE201382. 


\section{PTERULACEAE}

*Deflexula cf. major Corner, Annals of Botany 16:277 (1952). IMBABURA: Cantón Cotacachi. García Moreno. Reserva Los Cedros. 15-enero-2011. B. Dentinger, T.S. Jenkinson y J. McAlpine 809. QCNE201393.

*Deflexula cf. subsimplex (Henn.) Corner, Annals of Botany 16: 279 (1952). ORELLANA: Cantón: Aguarico. Tiputini field station. 18-julio-2004. T. Laessoe, K. Hansen, J.H. Petersen y A. Alsgard Jensen 11525. QCNE186329.

*Pterula brunneosetosa Corner, Annals of Botany 16 (4): 566 (1952). CARCHI: Cantón San Pedro de Huaca. Parroquia Mariscal Sucre. Estación Biológica Guandera. 24-julio-2004. Laessoe, J.H Petersen y C. Cárdenas-Batallas, 11639. QCNE186506.

*Pterula echo D.J. McLaughlin \& E.G. McLaughlin, Canadian Journal of Botany 58 (12): 1328 (1980). IMBABURA: Cantón Cotacachi. García Moreno. Reserva Los Cedros. 20-enero-2010. M. Wherler, J. McAlpine y B. Roy 240. QCNE201372.

*Pterula robusta Corner, Proceedings of the Linnean Society London 178: 101 (1967). IMBABURA: Cantón Cotacachi. García Moreno. Reserva Los Cedros. 20-enero-2010. M. Wherler, J. McAlpine y B. Roy 273. QCNE201374.

*Pterula secundiramea (Lév.) Speg., Boln Acad. nac. Cienc. Córdoba 11(4): 466 (1889). PICHINCHA: Cantón Quito. Nono. Reserva Orquideológica "El Pahuma". Km 43 vía CalacalíLa Independencia. 24-septiembre-2004. M. Villegas, C. Padilla y R. Leyva 2510. QCNE 244741.

\section{SCHIZOPHYLLACEAE}

Schizophyllum commune Fr., Observ. mycol. (Havniae) 1: 103 (1815). COTOPAXI: Cantón Sigchos. Parroquia Huacusi, Propiedad Fmlia. Caisapanta Maldonado, cerca del río Toachi.12-agosto-1998. D. Suárez 12. QCNE201192.

*Schizophyllum fasciatum Pat., J. Bot., Paris 1: 170 (1887). QCNE186946. PICHINCHA: Cantón Mejía. Uyumbicho. Refugio de Vida Silvestre Pasochoa, sendero rojo. 11-mayo2005. F. Salazar 88. QCNE186946.

\section{STROPHARIACEAE}

*Deconica coprophila (Bull.) P. Karst., Bidr. Känn. Finl. Nat. Folk 32: 515 (1879). QCNE201428. IMBABURA: Cantón Cotacachi. García Moreno. Reserva Los Cedros. 15-mayo-2014. R. Vandegrift, D. Newman y S. Boden 1396. QCNE201428.

Hypholoma fasciculare (Huds.) P. Kumm., Führ. Pilzk. (Zerbst): 72 (1871). QCNE186281. ORELLANA: Cantón: Aguarico. Tiputini field station. 18-julio-2004. Laessoe, K. Hansen, J.H. Petersen y A. Alsgard Jensen 11518. QCNE186281.

\section{TRICHOLOMATACEAE}

Collybia syringea Singer, Mycologia 47(5): 768(1955). MANABÍ: Cantón Puerto López, Rio Ayampe near the village. 31-julio-2004. T. Laessoe, K Hansen, J.H Petersen y A. Alsgard 11735. QCNE186443.

*Collybia tuberosa (Bull.) P. Kumm., Führ. Pilzk. (Zerbst): 119 (1871). CARCHI: Cantón San Pedro de Huaca. Parroquia Mariscal Sucre. Estación Biológica Guandera. 24-julio-2004. D. Suárez 513. QCNE152270. 
*Phyllotopsis cf. subnidulans (Overh.) Singer, Beih. Botan. Centralbl., Abt. B 56: 143 (1936). CARCHI: Cantón Espejo. Zona de amortiguamiento de la Reserva Ecológica "El Ángel", sector El Voladero. 1-octubre-2004. M. Villegas, T. Sanjuán, C. Padilla y R. Leyva 2532. QCNE201121

\section{TYPHULACEAE}

*Macrotyphula aff. rhizomorpha R.H. Petersen, Bull. N.Z. Dept. Sci. Industr. Res., PI. Dis. Div. 236: 80 (1988). PICHINCHA: Cantones: Quito y Mejía. Reserva Biológica Río Guajalito. Km 59 de la vía antigua Quito - Sto.Domingo. 15-octubre-2004. M. Villegas 2567. QCNE201092.

\section{AURICULARIALES \\ AURICULARIOMYCETIDAE}

Pseudohydnum gelatinosum (Scop.) P. Karst., Not. Sällsk. Fauna et Fl. Fenn. Förh. 9: 374 (1868). ZAMORA CHINCHIPE: Cantón Zamora. El tambo. Estación Científica San Francisco carretera Loja-Zamora. D. Suárez 1005. QCNE152737.

\section{AURICULARIACEAE}

Auricularia auricula-judae (Bull.) Quél., Enchir. fung. (Paris): 207 (1886). PICHINCHA: Cantón Quito. Nanegal. Zona Gualea, Finca Mapali. 6-agosto-2000. D. Suárez y M. Arévalo 302 QCNE201224.

Auricularia cornea Ehrenb., Horae Phys. Berol: 91 (1820). PICHINCHA: Cantón Quito. Nanegal. Zona Gualea, Finca Mapali. 22-febrero-2001. W. Chamorro, D. Montalvo, A. Luzuriaga y M. Ortega 85. QCNE2152172.

Auricularia delicata (Mont. ex Fr.) Henn., Bot. Jb. 17: 492 (1893). PICHINCHA: Cantón Santo Domingo de los Colorados, km 11 1/2 vía Quevedo. Estación Biológica Gustavo Orces. 4-diciembre-1999. D. Suárez, G. Pérez y M. Trujillo 222. QCNE152237.

Auricularia fuscosuccinea (Mont.) Henn., Bot. Jb. 17: 19 (1893). PICHINCHA: Cantón Santo Domingo de los Colorados, km 11 1/2 vía Quevedo. Estación Biológica Gustavo Orcés. 2-junio-1999. D. Suárez, G. Pérez y M. Trujillo 226. QCNE152818.

Auricularia mesenterica (Dicks.) Pers., Mycol. eur. (Erlanga) 1: 97 (1822). PICHINCHA: Cantón Quito. Nanegal. Zona Gualea, Finca Mapali. 22-febrero-2001. W. Chamorro, D. Montalvo, A. Luzuriaga y M Ortega 123. QCNE2152251.

Auricularia nigricans (Sw.) Birkebak, Looney \& Sánchez-García, N. Amer. Fung. 8(6): 12 (2013). IMBABURA: Cantón Cotacachi. García Moreno. Reserva Los Cedros. 15-enero-2008. B. Dentinger y T. S. Jenkinson 57. QCNE201361.

\section{BOLETALES}

\section{BOLETINELLACEAE}

Boletinellus exiguus (Singer \& Digilio) Watling, Edinburgh Journal of Botany 54: 243 (1997). PICHINCHA: Cantón Quito. Nanegalito, sector Pachijal Km 69 vía Calacalí - Los Bancos. 14-julio-2001. R. Batallas y 4 "G" Ecoturismo. Proyecto Ecoturistico MANA RIKSISHKA URKU 470. QCNE152806. 
Boletinellus monticola (Singer) Watling, Edinb. J. Bot. 54(2): 245 (1997). NAPO: Cantón Quijos. Parroquia Papallacta, Below Papallacta, just in disturbed river bank. 2-agosto-2004. T. Laessoe, K. Hansen, J.H Petersen y A. Alsgard 11758. QCNE186548.

Sclerodermataceae

*Scleroderma areolatum Ehrenb., Sylv. mycol. berol. (Berlin): 27 (1818). PICHINCHA: Cantón Quito. Bosque Protector Pichincha, SE de Quito, Loma el Ungui, Ciudadela Tarqui. 7-junio-2003. R. Batallas, C. Padilla y I. Jalca 544. QCNE152879.

\section{SUILLACEAE}

Suillus luteus (L.) Roussel, Fl. Calvados: 34 (1796). ZAMORA CHINCHIPE: Cantón Zamora. El Tambo, Estación Científica San Francisco carretera Loja-Zamora. 10-septiembre-2001. D. Suárez 543. QCNE152588.

\section{CANTHARELLALES \\ APHELARIACEAE}

*Aphelaria cf. tropica (Mont.) Corner, Monograph of Clavaria and allied Genera, (Annals of Botany Memoirs No. 1): 191 (1950). ORELLANA: Cantón: Aguarico. Tiputini field station. 14-julio-2004. T. Laessoe, J.H. Petersen y A. Alsgard Jensen 11434. QCNE186361.

\section{HYDNACEAE}

*Craterellus cornucopioides (L.) Pers., Mycol. eur. (Erlanga) 2: 5 (1825). ZAMORA CHINCHIPE: Cantón Nangaritza, Rio Nangaritza. Shaime. Confluencia de los rios Nangaritza y Numpatakaime. 15-octubre-1991. E. Freire 13. QCNE 152629.

\section{DACRYMYCETALES \\ DACRYMYCETACEAE}

Calocera viscosa (Pers.) Fr., Syst. mycol. (Lundae) 1: 486 (1821). CARCHI: Cantón San Pedro de Huaca. Parroquia Mariscal Sucre. Estación Biológica Guandera, sendero Oso cerca de la casa de Estación. 14-junio-2002. C. Padilla y A. Alvarez 513. QCNE 186215.

Dacryopinax spathularia (Schwein.) G.W. Martin, Lloydia 11: 116 (1948). PICHINCHA: Cantón Quito. Bosque Protector Pichincha. Sector Bosque Rosa de los Andes. 10-mayo2003. T. Sanjuán, C. Padilla y R. Batallas 529. QCNE 152751.

*Dacrymyces chrysospermus Berk. \& M.A. Curtis, Grevillea 2(no. 14): 20 (1873). IMBABURA: Cantón Cotacachi. Sector Plaza Gutiérrez. Intag. 23-agosto-2004. G. Romero 76. QCNE186985. Guepiniopsis alpina (Earle) Brasf. Am. Midl. Nat. 20: 225 (1938). CARCHI: Cantón San Pedro de Huaca. Parroquia Mariscal Sucre. Estación Biológica Guandera, sendero largo desde las turberas de frailejones. 21-marzo-2009. C.D, O.L.A y M. Bernal 115. QCNE244825.

\section{GEASTRALES}

\section{GEASTRACEAE}

Geastrum mirabile Mont., Crypt. Guyan. 595 (1855). PICHINCHA: Cantón Quito. Nanegal. Zona Gualea, Finca Mapali. 18-agosto-2001. W. Chamorro, D. Montalvo, A. Luzuriaga y M. Ortega 130. QCNE152851. 
Geastrum cf. triplex Jungh., Tijdschr. Nat. Gesch. Physiol. 7: 287 (1840). IMBABURA: Cantón Cotacachi. García Moreno. Reserva Los Cedros. 17-junio-2011. B. Dentinger y T.S. Jenkinson 821. QCNE201392.

*Myriostoma coliforme (Dicks.) Corda, Anleit. Stud. Mykol., Prag: LXXXI (1842). CARCHI: Cantón San Pedro de Huaca. San Gabriel. 25-julio-2004. T. Laessoe, J.H Petersen y C. Cárdenas Batallas 11694. QCNE186575.

\section{GOMPHALES \\ GOMPHACEAE}

*Phaeoclavulina cyanocephala (Berk. \& M.A. Curtis) Giachini, Mycotaxon 115: 191 (2011). IMBABURA: Cantón Cotacachi. García Moreno. Reserva Los Cedros. 18-junio-2011. B. Dentingen y T. S. Jenkinson 830. QCNE201394

*Phaeoclavulina aff. decurrens (Pers.) J.H. Petersen, Index Fungorum 369: 1 (2018). PICHINCHA: Cantón Santo Domingo de los Colorados. Sector finca "La Chamarra", vía Sto. Domingo-Quininde Km. 19-enero-2002. R. Batallas 212. QCNE152328.

*Ramaria cf. grandis (Peck) Corner, Monograph of Clavaria and allied Genera, (Annals of Botany Memoirs No. 1): 595 (1950). PICHINCHA: Cantones: Quito y Mejía. Bosque Protector Río Guajalito (QUSF). 14-octubre-2004. K. Escobar 52. QCNE186763.

\section{HYMENOCHAETALES}

*Trichaptum trichomallum (Berk. \& Mont.) Murrill, Bull. Torrey bot. Club 31(11): 608 (1904). PICHINCHA: Cantón Quito. Nanegal. Zona Gualea, Parche B, noreste $67^{\circ}$ de Gualea Cruz. 06-agosto-2000. D. Suárez y M. Arévalo 295. QCNE152497.

\section{HYMENOCHAETACEAE}

Hymenochaete rubiginosa (Dicks.) Lév., Annls Sci. Nat., Bot., sér. 3 5: 150 (1846). CARCHI: Cantón San Pedro de Huaca. Parroquia Mariscal Sucre. Estación Biológica Guandera. 25-agosto -2004. V. Jácome 91A. QCNE201501.

Inonotus aff. pseudoradiatus (Pat.) Ryvarden, Occ. Pap. Farlow Herb. Crypt. Bot. 18: 30 (1983). CARCHI: Cantón San Pedro de Huaca. 25-julio-2004. T. Laessoe, J.H. Petersen y C. Cárdenas- Batallas, K. 11699. QCNE186576.

*Inonotus tabacinus (Mont.) G. Cunn., Bull. N.Z. Dept. Sci. Industr. Res., Pl. Dis. Div. 78: 3 (1948). NAPO: Cantón Quijos. Parroquia Papallacta, shrub along small river above" aguas termales". 22-julio-2004. T. Laesseo, J.H. Petersen y C. Cárdenas Batallas11568. QCNE 186467.

Phylloporia spathulata (Hook.) Ryvarden, Syn. Fung. (Oslo) 5: 196 (1991). PICHINCHA: Cantón San Miguel de los Bancos. Parroquia Mindo. Reserva Mindo Lindo. 22-agosto-2001. D. Suárez, P. Aguirre y C. Garzón 610. QCNE152465.

\section{SCHIZOPORACEAE}

*Xylodon sambuci (Pers.) Țura, Zmitr., Wasser \& Spirin, Biodiversity of Cyanoprocaryotes, Algae and Fungi of Israel (Ruggel): 221 (2011). PICHINCHA: Cantón Quito. Bosque Protector Pichincha, SE de Quito, Loma el Ungui, Ciudadela Tarqui. 7-junio-2003. C. Padilla, R. Batallas y I. Jalca 62. QCNE152788. 


\section{PHALLALES \\ PHALLACEAE}

*Mutinus bambusinus (Zoll.) E. Fisch., Ann. Jard. Bot. Buitenzorg 6: 30 (1887). ORELLANA: Cantón: Aguarico. Tiputini field station. 18-julio-2004. T. Laessoe, K. Hansen, J.H. Petersen y A. Alsgard Jensen s/n. QCNE186582.

*Staheliomyces cinctus E. Fisch., Mitt. Naturf. Ges. Bern: 142 (1921) [1920]. PICHINCHA: Cantón Pedro Moncayo. Malchinguí. Bosque Protector Jerusalem, sendero avifauna. 7-mayo-2006. R. Batallas y A. Troya 1128. QCNE186878.

\section{POLYPORALES \\ FOMITOPSIDACEAE}

Laetiporus sulphureus (Bull.) Murrill, Mycologia 12(1): 11 (1920). QCNE201601. PICHINCHA: Cantón San Miguel de los Bancos. Parroquia Mindo. Los Bancos km 79 de la carretera Calacali-La Independencia. Bosque Protector Mindo Lindo. 1-septiembre-2001. D. Suárez, C. Cárdenas y L. Pinos 684.QCNE201065.

*Wolfiporia cocos (Schwein.) Ryvarden \& Gilb. Mycotaxon 19: 141 (1984). PICHINCHA: Cantón Santo Domingo de los Colorados, km 11 1/2 vía Quevedo. Estación Biológica Gustavo Orcés. 04-diciembre-1999. D. Suárez y M. Arévalo 204. QCNE152564.

\section{GANODERMATACEAE}

*Amauroderma camerarium (Berk.) J.S. Furtado, Revisâo do gênero Amauroderma (Polyporaceae); Estudos baseados nas microestruturas do basidiocarpo: 140 (1968). ORELLANA: Cantón: Aguarico. Tiputini field station. 16-julio-2004. T. Laessoe y J. H. Petersen 11489. QCNE186268.

Ganoderma cf. applanatum (Pers.) Pat., Hyménomyc. Eur. (Paris): 143 (1887). IMBABURA: Cantón Cotacachi. García Moreno. Reserva Los Cedros. 2-febrero-2008. B. Dentinger y T.S. Jenkinson 140. QCNE201386.

Ganoderma australe (Fr.) Pat., Bull. Soc. mycol. Fr. 5(2,3): 65 (1889). NAPO: Cantón Tena. Estación Biológica Jatun Sacha, a $8 \mathrm{Km}$ al este de Misahualli, al margen derecho del río Napo 2-enero-2001. D. Suárez 310. QCNE152316

Ganoderma cf. lucidum (Curtis) P. Karst., Revue mycol., Toulouse 3(no. 9): 17 (1881). ORELLANA: Cantón: Aguarico. Tiputini field station. 16-julio-2004. T. Laessoe, J.H Petersen y A. Alsgard Jensen 11476. QCNE186419.

\section{IRPICACEAE}

*Irpex lacteus (Fr.) Fr., Elench. fung. (Greifswald) 1: 142 (1828). PICHINCHA: Cantón Quito. Nanegal. Zona Gualea, Finca Mapali. 18-agosto-2001. D. Suárez 249. QCNE152339.

\section{MERIPILACEAE}

*Hydnopolyporus fimbriatus (Cooke) D.A. Reid, Persoonia 2(2): 151 (1962). PICHINCHA: Cantón Quito. Nanegalito. SE of Nanegalito, km 49, Puma Sacha, cascada del rayo. 10-julio-2004. T. Laessoe, J.H Petersen, C. A Padilla y R. Batallas 11360. QCNE 186366. 
*Hydnopolyporus palmatus (Hook.) O. Fidalgo, Mycologia 55(6): 715 (1963). PICHINCHA: Cantón Santo Domingo de los Colorados, km 11 1/2 vía Quevedo. Estación Biológica Gustavo Orcés. 4-diciembre-1999. D. Suárez, G. Pérez y M. Trujillo 209. QCNE152330.

Rigidoporus microporus (Sw.) Overeem, Icon. Fung. Malay. 5: 1 (1924). ORELLANA: Cantón Loreto. Faldas del Volcán Sumaco, a 2 km al oeste del río Huatarac. 21-marzo-1996. Efraín Freire 06. QCNE152610.

Rigidoporus vinctus (Berk.) Ryvarden, Norw. Jl. Bot. 19(2): 143 (1972). PICHINCHA: Cantón Quito. Nanegalito, SE of Nanegalito, km 49, Puma Sacha, cascada del rayo. 10-julio-2004. T. Laessoe, J.H Petersen, C. A Padilla y R. Batallas 11337. QCNE186347.

\section{MERULIACEAE}

Coralloderma nigripes (D.A. Reid) A.L. Welden, Fl. Neotrop., Monogr. 106: 19 (2010). ORELLANA: Cantón: Aguarico. Tiputini field station. 14-julio-2004. T. Laessoe, J.H Petersen y A. Alsgard Jensen 11438. QCNE186263.

Cymatoderma caperatum (Berk. \& Mont.) D.A. Reid, Kew Bull. [10]: 635 (1956) [1955]. PICHINCHA: Cantón Santo Domingo de los Colorados. Sector finca "La Chamarra", vía Sto. Domingo-Quininde Km. 19-enero-2002. R. Batallas 253. QCNE152277.

Flaviporus liebmannii (Fr.) Ginns, Can. J. Bot. 58(14): 1584 (1980). PICHINCHA: Cantón San Miguel de los Bancos. Parroquia Mindo. Los Bancos km 79 de la carretera CalacaliLa Independencia. Bosque Protector Mindo Lindo. 1-octubre-2001. D. Suárez y Colaboradores 773. QCNE201037.

*Hydnophlebia chrysorhiza (Eaton) Parmasto, Eesti NSV Tead. Akad. Toim., Biol. seer 16(4): 384 (1967). IMBABURA: Cantón Cotacachi. García Moreno. Reserva Los Cedros. 23-abril-2014. R. Vandegrift y D. Newman 1348. QCNE201426.

*Hyphoderma cf. nudicephalum Gilb. \& M. Blackw., Mycotaxon 33: 378 (1988). PICHINCHA: Cantón Quito. Bosque Protector Pichincha. Sector Bosque Rosa de los Andes. 10-mayo2003. C. Padilla, T. Sanjuán y R. Batallas 549. QCNE152770.

Phlebia tremellosa (Schrad.) Nakasone \& Burds., Mycotaxon 21: 245 (1984). IMBABURA: Cantón Cotacachi. García Moreno. Reserva Los Cedros. 14-abril-2014. R. Vandegrift y D. Newman 1231. QCNE201416.

*Podoscypha cristata (Berk. \& M.A. Curtis) D.A. Reid, Beih. Nova Hedwigia 18: 174 (1965). IMBABURA: Cantón Cotacachi. García Moreno. Reserva Los Cedros. 15-enero-2008. B. Dentinger y T.S. Jenkinson 56. QCNE201358.

\section{POLYPORACEAE}

*Cerrena caperata (Berk.) Zmitr, Mycena 1(1): 91 (2001). PICHINCHA: Cantón San Miguel de los Bancos. Parroquia Mindo. Bosque Protector Mindo Lindo. 4-febrero-2001. E. Muela y R. Batallas 06. QCNE152543.

Cerrena hydnoides (Sw.) Zmitr, Mycena 1(1): 91 (2001). PICHINCHA: Cantón Quito. Nanegal. Zona Gualea, Finca Mapali. 6-agosto-2000. W. Chamorro, D. Montalvo, A. Luzuriaga y M. Ortega 81. QCNE152491.

Cerioporus varius (Pers.) Zmitr. \& Kovalenko, International Journal of Medicinal Mushrooms (Redding) 18(1): 33 (2016). [MB\#812042]. PICHINCHA: Cantón Quito. Nanegal. Zona Gualea Cruz. 6-agosto-2000. D. Suárez y M. Arévalo 282. QCNE152412. 
Earliella scabrosa (Pers.) Gillb. \& Ryvarden, Mycotaxon 22(2): 364 (1985). NAPO: Cantón Tena. Estación Biológica Jatun Sacha, a $8 \mathrm{Km}$ de puerto Misahualli margen derecha del rio Napo. 16-septiembre-2002. R. Batallas, A. Mariscal y L. Parra 395. QCNE186094.

*Favolus aff. alveolarius (Bosc) Quél., Syst. orb. veg. (Lundae): 185 (1886). PICHINCHA: Cantón Quito. Nanegal. Zona Gualea, Finca Mapali. 14-diciembre-1999. W. Chamorro, D. Montalvo, A. Luzuriaga y M. Ortega 71. QCNE152884.

Favolus tenuiculus P. Beauv., Flore Oware Benin: 74 (1806). PICHINCHA: Cantón Quito. Nanegal. Zona Gualea, Finca Mapali. 6-agosto-2000. D. Suárez y M. Arévalo. 298. QCNE 152305.

*Fomes extensus (Lév.) Cooke, Grevillea 14(69): 18 (1885). PICHINCHA: Cantón Santo Domingo de los Colorados, km 11 1/2 vía Quevedo. Estación Biológica Gustavo Orces. 4-diciembre-1999. D. Suárez, G. Pérez y M. Trujillo 197. QCNE201051.

*Fomes fasciatus (Sw.) Cooke, Grevillea 14(69): 21 (1885). PICHINCHA: Cantón Quito. Nanegal. Zona Gualea, Finca Mapali. 22-febrero -2001. W. Chamorro, D. Montalvo, A. Luzuriaga y M. Ortega 73. QCNE152312.

*Hapalopilus rutilans (Pers.) Murrill, Bull. Torrey bot. Club 31 (8): 416 (1904). PICHINCHA: Cantón Quito. Nanegal. Zona Gualea, Finca Mapali. 6-agosto-2000. D. Suárez y M. Arévalo. 299. QCNE 201225.

Lentinus arcularius (Batsch) Zmitr., International Journal of Medicinal Mushrooms (Redding) 12(1): 88 (2010). PICHINCHA: Cantón Quito. Nanegal. Zona Gualea, Finca Mapali. 10-agosto-2001. W. Chamorro, D. Montalvo, A. Luzuriaga y M. Ortega 107. QCNE152480. Lentinus badius (Berk.) Berk., London J. Bot. 6: 491 bis (1847). SUCUMBIOS: Cantón Dorado de Cascales. Campo Bermejo. 21-abril-2001. D. Suárez 380. QCNE152440.

*Lentinus aff. brumalis (Pers.) Zmitr., International Journal of Medicinal Mushrooms (Redding) 12(1): 88 (2010). PICHINCHA: Cantón Quito. Nanegal. Zona Gualea, Finca Mapali. 10-agosto-2001. W. Chamorro, D. Montalvo, A. Luzuriaga y M. Ortega 67. QCNE152481.

*Lentinus aff. copulatus (Ehrenb.) Henn., Nat. Pflanzenfam., Teil. I (Leipzig) 1(1): 224 (1898) [1900]. ORELLANA: Cantón Aguarico. Tiputini field station. 16-julio-2004. T. Laessoe, J.H Petersen, y A. Alsgard Jensen, C.A 11459. QCNE186291.

Lentinus crinitus (L.) Fr., Syst. orb. veg. (Lundae) 1: 77 (1825). PICHINCHA: Cantón Quito. Nanegal. Zona Gualea Cruz. 5-agosto-2000. D. Suárez y M. Arévalo. 245. QCNE152451.

Lentinus tricholoma (Mont.) Zmitr., International Journal of Medicinal Mushrooms (Redding) 12 (1): 88 (2010). ORELLANA: Cantón Aguarico. Tiputini field station. 18-agosto-2004. T. Orellana. Bataburo Lodge, orillas del río Tiguino en territorio Huaorani. L. Guzmán Dávalos 9215. QCNE186212.

*Lentinus velutinus Fr., Linnaea 5: 510 (1830). NAPO: Cantón Tena. Estación Biológica Jatun Sacha, a $8 \mathrm{Km}$ al este de Misahualli, al sur del del río Napo. 16-septiembre-2002. R. Batallas, A. Mariscal y L. Parra 382. QCNE186084.

*Nigroporus vinosus (Berk.) Murrill, Bull. Torrey bot. Club 32(7): 361 (1905). IMBABURA: Cantón Cotacachi. García Moreno. Reserva Los Cedros. 15-junio-2008. B. Dentinger y T. S. Jenkinson 65. QCNE201360.

Picipes badius (Pers.) Zmitr. \& Kovalenko, International Journal of Medicinal Mushrooms (Redding) 18(1): 35 (2016). PICHINCHA: Cantón Quito. Orquideológica El Pahuma, km 22 vía Calacalí - La Independencia. 2-noviembre-1998. D. Suárez, J. Quezada y M. Díaz 87. QCNE152482.

Polyporus cf. citrinus Pers., Mycol. eur. (Erlanga) 2: 49 (1825). PICHINCHA: Cantón San Miguel de los Bancos. Parroquia Mindo. Bosque Protector Mindo Lindo. Km 79 de la carretera Calacali. 1-agosto-2001. D. Suárez y Colaboradores 615. QCNE201022. 
Polyporus dictyopus Mont., Annls Sci. Nat., Bot., sér. 2 3: 349 (1835). IMBABURA: Cantón Cotacachi. García Moreno. Reserva Los Cedros. 7-febrero-2010. J. McAlpine 665. QCNE201381. Polyporus leprieurii Mont., Annls Sci. Nat., Bot., sér. 2 13: 203 (1840). PICHINCHA: Cantón San Miguel de los Bancos. Parroquia Mindo. Bosque Protector Mindo Lindo. Km 79 de la carretera Calacalí. 1-agosto-2001. D. Suárez y Colaboradores 619. QCNE201038.

Pseudofavolus tenuis (Fr.) G. Cunn., Bull. N.Z. Dept. Sci. Industr. Res. 164: 185 (1965). SUCUMBIOS: Cantón Shushufindi, Parroquia Limoncocha. Reserva Biológica Limoncocha, sendero Pishira. 30-julio-2001. C. Cárdenas, P. Gamboa y L. Pinos 25. QCNE186175.

Pycnoporus sanguineus (L.) Murrill, Bull. Torrey bot. Club 31(8): 421 (1904). ZAMORA CHINCHIPE: Cantón Nangaritza. Rio Nangaritza. Shaime. Confluencia de los Rios Nangaritza y Numoatakaime.15-octubre-1991. E. Freire10. QCNE152572.

*Skeletocutis nivea (Jungh.) Jean Keller, Persoonia 10 (3): 353 (1979). PICHINCHA: Cantón Quito. Nanegalito, SE of Nanegalito, km 49, Puma Sacha, cascada del rayo. 10-julio-2004. T. Laessoe, J.H Petersen, C. Padilla \& R. Batallas 11355. QCNE186358.

Tinctoporellus epimiltinus (Berk. \& Broome) Ryvarden, Trans. Br. mycol. Soc. $73(1)$ : 18 (1979). IMBABURA: Cantón Cotacachi. García Moreno. Reserva Los Cedros. 11-abril-2014. R. Vandegrift y D. Newman 1219. QCNE201415.

Trametes elegans (Spreng.) Fr., Epicr. syst. mycol. (Upsaliae): 492 (1838) [1836-1838]. IMBABURA: Cantón Cotacachi, Sector Plaza Guitiérrez. Intag. Protector San Luis de Toabuche.17-julio-2001. N. Chasiliquin y A. Troya 049. QCNE152347.

Trametes membranacea (Sw.) Kreisel, Monografias, Ciencias, Univ. Habana, Ser. 4 16: 83 (1971). IMBABURA: Cantón Cotacachi. García Moreno. Reserva Los Cedros. 24-enero-2010. B. Roy 409. QCNE201375.

Trametes versicolor (L.) Lloyd, Mycol. Notes (Cincinnati) 65: 1045 (1921) [1920]. PICHINCHA: Cantón Quito. Bosque Protector Pichincha. Sector Bosque Cochapamba. 3-marzo-2003. R. Batallas, T. Sanjuán, C. Padilla 520. QCNE152511.

Trametes villosa (Sw.) Kreisel, Monografias, Ciencias, Univ. Habana, Ser. 4 16: 83 (1971). PICHINCHA: Cantón San Miguel de los Bancos. Parroquia Mindo. Santuario de las cascadas. 17-julio-2001. R. Batallas 179. QCNE152324.

\section{RUSSULALES}

*Laeticutis cristata (Schaeff.) Audet, Mycotaxon 111: 442 (2010). PICHINCHA: Cantón Quito. Nanegal. Zona Gualea, Finca Mapali. 22-febrero-2001. W. Chamorro, D. Montalvo, A. Luzuriaga y M. Ortega 76. QCNE152484.

\section{ALBATRELLACEAE}

*Albatrellopsis flettii (Morse ex Pouzar) Audet, Mycotaxon 111: 440 (2010). PICHINCHA: Cantón Quito. Nanegal. Zona Gualea, Finca Mapali. 10-agosto-2001. W. Chamorro, D. Montalvo, A. Luzuriaga y M. Ortega 120. QCNE152884.

\section{AURISCALPIACEAE}

Artomyces pyxidatus (Pers.) Jülich, Biblthca Mycol. 85: 399 (1982) [1981]. CARCHI: Cantón San Pedro de Huaca. Parroquia Mariscal Sucre. Estación Biológica Guandera. 3-diciembre-2002. R. Batallas, T. Sanjuán y C. Padilla 441. QCNE186768. 
*Artomyces turgidus (Lév.) Jülich, Biblthca Mycol. 85: 399 (1982) [1981]. ZAMORA CHINCHIPE: Cantón Zamora. El Tambo, Estación Científica San Francisco carretera LojaZamora. 28-marzo-2002. D. Suárez 1019. QCNE152264.

*Auriscalpium cf. andinum (Pat.) Ryvarden, Harvard Pap. Bot. 6(1): 194 (2001). ORELLANA: Cantón Aguarico. Tiputini field station. 13-julio-2004. T. Laessoe, K. Hansen, J.H. Petersen y A. Alsgard Jensen 11414. QCNE 186478.

Auriscalpium villipes (Lloyd) Snell \& E.A. Dick, Lloydia 21: 35 (1958). IMBABURA: Cantón Cotacachi. Sector Plaza Guitiérrez. Intag. Protector San Luis de Toabuche. 17-julio-2001. N. Chasiliquin y A. Troya 055. QCNE152331.

Auriscalpium vulgare Gray, Nat. Arr. Brit. Pl. (London) 1: 650 (1821). PICHINCHA: Cantón San Miguel de los Bancos. Parroquia Mindo. Km 85 vía Quito-Mindo. 29-agosto-2005. N. Baño 58. QCNE201539.

\section{LACHNOCLADIACEAE}

${ }^{*}$ Asterostroma cf. laxum Bres., Bull. Soc. mycol. Fr. 36(1): 46 (1920). PICHINCHA: Cantón Quito. Volcán Pichincha. La primavera, N side of quebrada. 21-julio-2004. T. Laessoe, K. Hansen, J.H Petersen y A. Alsgard 11547. QCNE186561.

\section{STEREACEAE}

Stereum hirsutum (Willd.) Pers., Observ. mycol. (Lipsiae) 2: 90 (1800) [1799]. IMBABURA: Cantón Cotacachi. Sector Plaza Guitiérrez. Intag. Protector San Luis de Toabuche. 17-julio-2001. N. Chasiliquin y A. Troya 030. QCNE152536.

Stereum ostrea (Blume \& T. Nees) Fr., Epicr. syst. mycol. (Upsaliae): 547 (1838) [1836-1838. PICHINCHA: Cantón Quito. Bosque Protector Pichincha. Loma El Ungui, Ciudadela Tarqui. 7-junio-2003. C. Padilla, R. Batallas y I. Jalca 611. QCNE152751.

*Stereum cf. subtomentosum Pouzar, Ceská Mykologie 18 (3): 147 (1964). NAPO: Cantón Quijos. Just bellow Papallacta pass, páramo la virgen. T. Laessoe, K. Hansen, J.H. Petersen y A. Alsgard 11365. QCNE186274.

\section{STEREOPSIDALES STERIOPSIDACEAE}

Stereopsis radicans (Berk.) D.A. Reid. Beih, Nova Hedwigia 18: 314 (1965). PICHINCHA: Cantón San Miguel de los Bancos. Parroquia Mindo. Reserva Mindo Lindo. Los Bancos km 79 de la carretera Calacalí - La Independencia. 1-septiembre-2001. D. Suárez, C. Cárdenas y L. Pinos 704. QCNE201105.

\section{THELEPHORALES \\ THELEPHORACEAE}

Thelephora terrestris Ehrh., Pl. Crypt. Linn. Exsicc.: no. 178 (1793). PICHINCHA: Cantón Quito. Nanegal. Zona Gualea Cruz en la finca Mapali. 22-febrero-2001. W. Chamorro, D. Montalvo, A. Luzuriaga y M. Ortega 47. QCNE152591. 


\section{TRECHISPORALES HYDNODONTACEAE}

* Scytinopogon angulisporus (Pat.) Corner, Monograph of Clavaria and allied Genera, (Annals of Botany Memoirs No. 1): 648 (1950). ORELLANA: Cantón Aguarico. Tiputini field station. 13-julio-2004. T. Laessoe, K. Hansen, J.H. Petersen y A. Alsgard Jensen 11408. QCNE186254.

*Scytinopogon pallescens (Bres.) Singer, Lloydia 8: 139 (1945). QCNE186775.

\section{TREMELLALES \\ TREMELLACEAE}

*Tremella encephala Pers., Syn. meth. fung. (Göttingen) 2: 623 (1801). PICHINCHA: Cantón Quito. Nanegal. Zona Gualea Cruz. 5-agosto-2000. D. Suárez y M. Arévalo 264. QCNE152983.

*Tremella rufolutea Berk. \& M.A. Curtis, J. Linn. Soc., Bot. 10(no. 46): 340 (1868) [1869]. PICHINCHA: Cantón San Miguel de los Bancos. Parroquia Mindo. Trayecto del Oleoducto de Crudos Pesados OCP vía Nono - Tandayapa, Cerro Castillo, La Bola. 5-octubre-2001. D. Suárez 536. QCNE152985.

\section{DISCUSIÓN}

En la plataforma Fungi web Ecuador [28] se reporta para el país 843 especies entre hongos y hongos liquenizados. Considerando este dato la colección micológica del QCNE posee aproximadamente el 32\% de las especies de hongos reportadas para el país. Las familias más abundantes reportados en el Fungario QCA(M) de la PUCE, según Fungi Web, en Basidiomycota son: Polyporaceae (69 spp.), Marasmiaceae (35 spp.), Agaricaceae (32 spp.), Mycenaceae (29 spp.) y Meruliaceae (22 spp.) y en Ascomycota: Xylariaceae (122 spp.), Cordicipitaceae (20 spp.), Laboulbeniaceae (16 spp.), Pyronemataceae y Sarcoscyphaceae (9 spp.). En este artículo evidenciamos que las familias Polyporaceae (35 spp.), Marasmiaceae (14 spp.), Xylariaceae (33 spp.) y Cordycipitaceae (20 spp.) también son las más abundantes en el repositorio del QCNE. Los géneros mejor representados en el Fungario QCA(M) son: Ganoderma (19 spp.), Marasmius (16 spp.), Trametes (12 spp.) y Polyporus (12 spp.) en Basidiomycota y Xylaria (52 spp.), Camillea y Hypoxylon (18 spp.) y Cordyceps (11 spp.) en Ascomycota. En el repositorio del QCNE están mejor representados los géneros: Marasmius (7 spp.), Polyporus (6 spp.), Xylaria (33 spp.) y Cordyceps (19 spp.). Con este trabajo se contribuye con 144 especies al listado de Fungi web Ecuador [28].

En este análisis hay que considerar que falta ingresar y depurar un buen porcentaje de los especímenes depositados de proyectos de investigación con instituciones cooperantes que seguramente incrementarán el número de especímenes al repositorio. En la colección se reporta mayor número de basidiomicetes pertenecientes a hongos agaricales (hongos en sombrilla) y polyporales (poliporoides) pues la mayoría de colectas son muestreos en bosques montanos y subtropicales, y los hongos agaricales (en especial los miembros saprófitos) son considerados más diversos en latitudes bajas. Los polyporales 
son organismos frecuentes en bosques húmedos, cuyos factores cambiantes como: temperatura, pluviosidad y humedad relativa constituyen estrategias de adaptación que les permiten explotar el sustrato del medio y facilitan la dispersión de esporas [29].

La visita ocasional de especialistas en diferentes grupos taxonómicos ha permitido la revisión y determinación taxonómica de especímenes, pero al contrario la inexistencia de procesos como préstamos, intercambios y donaciones de duplicados con otros repositorios ha impedido el intercambio y actualización de información taxonómica. Ante esto, a futuro se pretende mejorar los procesos curatoriales de las muestras con la revisión de especímenes por parte de especialistas de los diferentes grupos.

La colección micológica del Herbario QCNE del INABIO constituye una fuente importante de referencia y base de datos sobre la biodiversidad de hongos, promoviendo el desarrollo de las actividades taxonómicas, divulgativas y de conservación de la funga del territorio ecuatoriano, además representa a nivel nacional el único repositorio micológico del sector gubernamental parte de un instituto público de investigación. A pesar de esto la diversidad y cantidad de colecciones de este repositorio público aún es muy baja y la información de importantes registros no está publicada.

\section{AGRADECIMIENTOS}

Expresamos nuestros agradecimientos a los estudiantes Jordi Rivera de la Universidad Tecnológica Indoamérica, Diego Córdova y Daniela Olmedo de la Universidad Central del Ecuador que como parte del programa de prácticas preprofesionales colaboraron en la digitalización y actualización de los datos en dicha colección. De igual forma a David Donoso (Escuela Politécnica Nacional) y a Nelson Miranda-Moyano (Universidad San Francisco de Quito) por las correcciones y recomendaciones realizadas al manuscrito en general.

\section{CONTRIBUCIÓN DE LOS AUTORES}

Rosa Batallas redactó el manuscrito y revisó los nombres del listado de especies en las plataformas correspondientes. Gabriela Moya analizó y tabuló los datos. Daniel Navas digitalizó la información para el análisis de los datos del manuscrito. 


\section{REFERENCIAS}

[1] Hokche, 0. y Rodríguez L. (2006). Herbario Nacional de Venezuela: El centro del conocimiento y conservación de la riqueza florística del país celebra 90 años. Museos.Ve, 6:10-13.

[2] Museo Ecuatoriano de Ciencias Naturales MECN. (1992). Serie: Revista Informativa Museo Ecuatoriano de Ciencias Naturales. 2(9). Quito.

[3] Base de Datos de Biodiversidad del Ecuador. 2020. Recuperado el 22 de junio de 2020 de: http://www.symbiota. org | https://bdj.pensoft.net/articles.php?id=1114 | https://github.com/Symbiota/

[4] Sanjuán, T., Tabima, J., Restrepo, A., Læssøe, S., Spatafora, T. y Franco-Molano, A. E. (2014). Entomopathogens of Amazonian stick insects and locust are members of the Beauveria species complex (Cordyceps sensu stricto). Mycologia, 106: 260-275. doi: https://doi.org/10.3852/106.2.260

[5] Sanjuán, T., Franco-Molano, A. E., Kepler, R. M., Spatafora, J. W., Tabima, J., Vasco-Palacios, A. M y Restrepo, S. (2015). Five new species of entomopathogenic fungi from the Amazon and evolution of neotropical Ophiocordyceps. Fungal Biology 1-16. doi: http:dx.doi.org/10.1016/j.funbio.2015.06.010

[6] Batallas, R. (2016). Caracterización morfológica y molecular de cuatro especies de hongos comestibles y medicinales en zonas de cultivo de la comunidad kichwa de Oglán - Pastaza, Ecuador (Tesis de maestría). Universidad de Guayaquil, Ecuador.

[7] Thomas, D., Vandegrift, R., Ludden, A., Carroll, G y Roy, B. (2016). Spatial Ecology of the Gungal Genus Xylaria in a Tropical Cloud Forest. Biotropica 48: 381-393. doi: https://doi.org/10.1111/btp.12273

[8] Lowy B. (1952). The genus Auricularia. Mycologia 44: 656-692.

[9] Dennis, R. W. G. (1982). Fungus Flora of Venezuela and adjacent countries. Fungus flora of Venezuela and adjacent countries. Royal Botanic Gardens, Kew. 125-135 pp.

[10] Gilbertson, R. L. y Ryvarden. L. (1987). North American polypores 2. Megasporoporia -Wrightoporia. Oslo: Fungiflora.

[11] Largent, D. y Baroni, T. (1988). How identify mushromms to genus VI: Modern Genera. Eureka, CA: Mad River Press.

[12] Ryvarden, L. (1991). Genera of polypores: Nomenclature and taxonomy. Synopsis Fungorum 5. Oslo: Fungiflora.

[13] Ryvarden, L. (2000). Studies in Neotropical polypores 8. Poroid fungi from Jamaica, a preliminar checklist. Mycotaxon 76: 349-360.

[14] Ryvarden, L. (2015a). Studies in Neotropical polypores 39 Trametes alba nova species. Synopsis Fungorum, 33:32-35.

[15] Ryvarden, L. (2015b). Clave taxonómica. Laetiporus - Lindtneria. Synopsis Fungorum. Oslo: Fungiflora

[16] Núñez, M. y Ryvarden. L. (1995). Polyporus (Basidiomycotina) and related genera. Synopsis Fungorum 10: 1-85.

[17] Mata, M. (1999). Macrohongos de Costa Rica. Costa Rica: INBio.

[18] Mata, M., Halling, R. y Mueller, G. (2003). Macrohongos de Costa Rica. Costa Rica: INBio.

[19] Franco-Molano, A. E., Vasco-Palacios A., López-Quintero, C. y Boekhout, T. (2005). Macrohongos de la Región del Medio Caquetá - Colombia. Medellín: Multimpresos.

[20] Wright, J., y Albertó, E. (2006). Guía de los Hongos de la Región Pampeana. Il Hongos sin laminillas. L.0.L.A:Argentina.

[21] Montoya-Alvarez, A. F., Hayakawa, H., Minamya, Y., Fukuda, T., López-Quintero, C. A., \& Franco-Molano, A. E. (2011). Phylogenetic relationships and review of the species of Auricularia (Fungi: Basidiomycetes) in Colombia. Caldasia 33(1), 55-66.

[22] Alvarenga, R. L., Naves L. R y Santos S. (2015). El género Auricularia Bull. Ex Juss. (Basidiomycota) en Cerrado (Sabana brasileña) áreas del estado de Goiás y el Distrito Federal. Mycosphere 6: 532-541. doi: https://doi. org/10.5943/mycosphere/6/5/3

[23] Index Fungorum. (2009). The Royal Botanic Gardens Kew, Landcare Research-NZ. Institute of Microbiology (Versión 3) Recuperado el 18 de julio de 2019 de: http://www.indexfungorum.org/names/Names.asp. 
[24] V. Robert, G. Stegehuis y J. Stalpers. (2005). The MycoBank engine and related databases. Recuperado el 7 de mayo de 2020 de: http://www.mycobank.org/quicksearch.aspx

[25] Rodríguez, 0., Herrera-Fonseca, M., Sánchez-Jácome, M. Del R., Álvarez, I., Valenzuela, R., Garć́a, J. y GuzmánDávalos, L. (2010). Catálogo de la micobiota del bosque La Primavera, Jalisco. Revista Mexicana de Micología, 2940. URL: http://www.scielo.org.mx/scielo.php?script=sci_arttext\&pid=S0187-31802010000200004\&lang=pt

[26] Dios, M. M., Albertó, E., y Moreno, G. (2011). Catálogo de hongos gasteroides (Basidiomycota) de Catamarca, Argentina. Bol. Soc. Argent. Bot, 46: 5-11.

[27] Fernández-Fernández, D., Freire, E., Peñafiel, M., Romero, G., Tello, F., y Toapanta, E. (2015). Catálogo de especímenes tipo del Herbario Nacional del Ecuador (QCNE). Museo Ecuatoriano de Ciencias Naturales. ACI Avances en Ciencias e Ingenierías 7(1): B39-B87. doi: https://doi.org/10.18272/aci.v7i1.227

[28] Ordoñez, M. E. (2018). Fungi del Ecuador. Versión 2018.0. Fungario QCAM, Pontificia Universidad Católica del Ecuador. Recuperado el 20 de enero de 2020 de: https:bioweb.bio/fungiweb

[29] Lodge, D. J., Ammirat, J. F., O' Dell, T. E., Mueller, G. M., Huhndorf , S. M., Wang, C., Stokland, J., Schmit, J. P., Ryvarden, L., Leacock, P., Mata, M., Umaña, L., Wu, Q., Czederpiltz, D. L. (2004). Terrestrial and lignicolous macrofungi. En Mueller, G., Bills, G., Foster, M. (Eds.), Biodiversity of fungi: inventory and monitoring methods (pp. 127-172). San Diego: Elsevier Inc. 